\title{
Friend or Foe: Hybrid proline-rich proteins determine how plants interact with and
} respond to beneficial and pathogenic microbes

3 Zeeshan Z. Banday ${ }^{1}$, Nicolás M. Cecchini ${ }^{1, \dagger}$, Allison T. $\operatorname{Scott}^{1}$, Ciara T. Hu ${ }^{1}$, Rachael C. Filzen ${ }^{1}$,

4 Elinam Agbo ${ }^{1}$, Jean T. Greenberg ${ }^{1, *}$

61 Department of Molecular Genetics and Cell Biology, The University of Chicago, 929 East 57th

7 Street GCIS W 524, Chicago, IL 60637, USA

$8 \uparrow$ Current address: Centro de Investigaciones en Química Biológica de Córdoba (CIQUIBIC),

9 CONICET, Departamento de Química Biológica Ranwel Caputto, Facultad de Ciencias

10 Químicas, Universidad Nacional de Córdoba, Córdoba, Argentina, Haya de la Torre y Medina

11 Allende, X5000HUA Córdoba, Argentina. Email: ncecchini@unc.edu.ar

13 *Correspondence: jgreenbe@uchicago.edu

15 Short title: HyPRPs control microbe induced growth and defense

16

17 The author responsible for distribution of materials integral to the findings presented in this 18 article in accordance with the policy described in the Instructions for Authors 19 (https://academic.oup.com/plcell/pages/General-Instructions) is: Jean T. Greenberg

20 (jgreenbe@uchicago.edu).

22 Keywords: HyPRP, systemic immunity, chloroplast targeting, root system architecture, biomass 


\section{Abstract}

26 Plant plastids generate signals, including some derived from lipids, that need to be mobilized to

27 effect signaling. We used informatics to discover potential plastid membrane proteins involved

28 in microbial responses. Among these are proteins co-regulated with the systemic immunity

29 component AZI1, a hybrid proline-rich protein (HyPRP) and HyPRP superfamily members.

30 HyPRPs have a transmembrane domain, a proline-rich region (PRR) and a lipid transfer protein

31 domain. The precise subcellular location(s) and function(s) is unknown for most HyPRP family

32 members. As predicted by informatics, a subset of HyPRPs have a pool of protein that targets

33 plastid outer envelope membranes (OEMs) via a mechanism that requires the PRR. Additionally,

34 two HyPRPs may be associated with thylakoid membranes. Most of the plastid and non-plastid

35 localized family members also have pools that localize to endoplasmic reticulum, plasma

36 membrane or plasmodesmata. HyPRPs with plastid pools regulate, positively or negatively,

37 systemic immunity against the pathogen Pseudomonas syringae. HyPRPs also regulate the

38 interaction with the plant growth promoting rhizobacteria Pseudomonas simiae WCS417 in the

39 roots to influence colonization, root system architecture and/or biomass. Thus, HyPRPs have

40 broad and distinct roles in immune, development and growth responses to microbes and reside at

41 sites that may facilitate signal molecule transport. 


\section{Introduction}

Plants have evolved strategies to provide immunity against a broad range of pathogens.

45 The recognition of pathogen-derived molecules at the surface or inside the cell leads to the

46 establishment of local or systemic immunity (Albert et al., 2020; Fu and Dong, 2013; Zipfel,

47 2008). During systemic immunity, the host plant responds to a primary infection to provide a

48 faster response against a broad range of secondary infections by a phenomenon called priming

49 (Jaskiewicz et al., 2011; Jung et al., 2009; Pieterse et al., 2014). The recognition of

50 pathogens/pathogen-derived molecules in the leaves induces systemic acquired resistance (SAR),

51 a broad spectrum program that suppresses diverse pathogens at sites distal to a primary infection

52 (Fu and Dong, 2013). Colonization of roots with beneficial bacteria can lead to induced-systemic

53 resistance (ISR) in the aboveground part of the plant (Pieterse et al., 2014). These systemic

54 immune programs require long distance transport of mobile signal(s) from the immunized tissue

55 (Carella, 2020; Cecchini et al., 2019b; Kachroo and Kachroo, 2020; Pieterse et al., 2014). Many

56 candidate signal molecules, including azelaic acid (AZA), have been identified as mobile signals

57 for SAR. These signals may act together to confer SAR (Jung et al., 2009; Kachroo and Kachroo,

58 2020). Although there are differences in the mechanism of induction, some of the signaling

59 components for SAR and ISR overlap (Cecchini et al., 2019b, 2015; Pieterse et al., 1996, 1998;

60 Beckers and Conrath, 2007; Beckers et al., 2009). Beneficial rhizobacteria not only induce ISR,

61 but also stimulate the growth and productivity of plants and impact the root system architecture

62 (Berendsen et al., 2012; Efthimiadou et al., 2020). A crosstalk between the signaling pathways of

63 rhizosphere-induced growth promotion and ISR remains unknown. However, some degree of

64 shared signaling seems plausible (Bulgarelli et al., 2013; Haney et al., 2015). 
Plastids coordinate many cellular responses to infections, generating signals that activate defenses (Bobik and Burch-Smith, 2015). Several key defense molecules including hormones

67 and second messengers are synthesized in plastids (Bobik and Burch-Smith, 2015; Dempsey et

68 al., 2011; Mittler et al., 2004). More importantly, signals needed for SAR, such as salicylic acid,

69 AZA and pipecolic acid are produced at least in part within plastids (Dempsey et al., 2011;

70 Hartmann et al., 2017; Zoeller et al., 2012; Rekhter et al., 2019). These molecules (or others

71 regulated by them) should be mobilized from their site(s) of synthesis for long-distance transport.

72 The SA precursor isochorismate synthesized in the chloroplasts is thought to be exported by

73 ENHANCED DISEASE SUSCEPTIBILITY5 (EDS5) (Rekhter et al., 2019; Serrano et al.,

74 2013). Similarly, pools of AZELAIC ACID INDUCED 1 (AZI1) and its paralog EARLY

75 ARABIDOPSIS ALUMINUM INDUCED 1 (EARLI1) are localized in the plastid envelopes and

76 help in the mobilization of plastid-produced AZA (Cecchini et al., 2015; Zoeller et al., 2012).

77 These proteins also localize to the endoplasmic reticulum (ER), and plasma membrane (PM),

78 including plasmodesmata (PD) (Cecchini et al., 2015). Pathogen infection or treatment with the

79 microbe (pathogen)-associated molecular pattern MAMP/PAMP flg22, an epitope of bacterial

80 flagellin, induces AZI1 and EARLI to become enriched at chloroplasts (Cecchini et al., 2015,

81 2020). The plastids together with other membranous organelles like nucleus, ER and PM

82 (including PD), facilitate the efficient relay of inter-organellar signaling to achieve whole-cell

83 immunity (Helle et al., 2013; Toulmay and Prinz, 2011; Caplan et al., 2015; Lee, 2015). AZI1

84 and EARLI1 are essential for SAR, MAMP/PAMP-triggered SAR, ISR and AZA-induced

85 systemic disease resistance (Cecchini et al., 2019b, 2015). AZI1 is a signal-anchored protein that

86 has an N-terminal bipartite signal composed of a signal peptide (SP)-like hydrophobic domain

87 followed by a charged protein region (CPR, consisting of at least three Lys and/or Arg residues, 
88 within eight amino acids at the C-terminal side of TMD) and proline rich region (PRR) for the

89 plastid targeting (Cecchini et al., 2015, 2020; Lee et al., 2011). The SP-like region acts as a

90 transmembrane domain (TMD) anchoring AZI1 to the membranes, whereas the PRR guides its

91 plastid association (Cecchini et al., 2020). The superfamily of proteins to which AZI1 belongs

92 are called hybrid proline-rich proteins (HyPRPs). Invariant features include the SP-like region

93 and a C-terminal 8-cysteine motif (8CM)/lipid transfer protein-like (LTP) domain. Most

94 members of the family also have a PRR between these regions that varies in length. Although

95 AZI1 uses a signal-anchored mechanism for plastid targeting, it does not conform to targeting

96 features of classical signal anchor proteins whose N-termini have low hydrophobicity scores on

97 the Wimley-White hydrophobicity scale (Lee et al., 2011).

In this work, we used bioinformatic analyses to identify proteins that, like AZI1, have a

99 pool of protein that localizes to plastid membranes, but which are not classical signal anchored

100 proteins. Included in the screen are proteins whose transcripts are co-regulated with AZI1 and

101 members of the HyPRP superfamily, respectively. Herein, we show the utility of our

102 bioinformatics approach for finding proteins with a pool that localizes to plastid membranes and

103 show that some of them, in the HyPRP superfamily, have different positive or negative roles in

104 systemic immunity, development and growth responses to microbes in roots and shoots.

105

106 Results

107 In silico analysis of subcellular targeting of the Arabidopsis proteome

108 Well-known targeting algorithms (TargetP, ChloroP and SignalP; (Emanuelsson et al., 109 1999, 2000; Nielsen et al., 1997) predict AZI1 to be a secreted protein. However, a pool of AZI1 110 (and that of close paralogs) localizes to plastid envelopes using an $\mathrm{N}$-terminal bipartite signal 
111 (SP-like/TMD + PRR; (Cecchini et al., 2015)) AZI1 has similar properties to apicomplexan

112 proteins that target apicoplasts (non-photosynthetic plastids) using N-terminal bipartite signal

113 sequences (Figure 1; (Cecchini et al., 2020)). The PATS algorithm, trained using apicomplexa

114 plastid proteins, can successfully predict plastid localization of AZI1 and paralogs by searching

115 for this bipartite signal (Cecchini et al., 2015, 2020; Zuegge et al., 2001). We used PATS to

116 predict N-terminal bipartite signal sequence and plastid localization of the total Arabidopsis

117 proteome. Overall, the PATS-generated prediction output (positive and negative selections)

118 showed that approximately $7-8 \%$ proteins coded by each chromosome may localize to plastids

119 (Figure 1B). The Arabidopsis proteins predicted to target to the plastids by PATS are listed in

120 Supplementary Dataset S1. Interestingly, the gene ontology (GO) functional characterization

121 (biological processes) of these proteins revealed that among others, the GO terms related to cell

122 communication, response to biotic stimulus, defense response, protein phosphorylation, cell wall

123 organization and cell-cell signaling were significantly overrepresented (Supplementary Figure

124 S1A).

125 To test our predictions, we selected six PATS-positive proteins whose transcripts showed

126 similar expression profiles and clustered together with AZI1 as assessed by the

127 GENVESTIGATOR ${ }^{\circledR}$ Hierarchical clustering tool (biological context: Perturbations; (Hruz et al.,

128 2008)). As a secondary screen, we chose proteins that did not conform to a key characteristic of

129 classical signal-anchored plastid OEM proteins. Specifically, their SP-like N-terminal regions

130 lacked low hydrophobicity scores $(<0.4)$ on the Wimley-White hydrophobicity scale (Lee et al.,

131 2011). Instead, the scores (calculated with the MPEx algorithm

132 (http://blanco.biomol.uci.edu/mpex; (Jayasinghe et al., 2001; Snider et al., 2009)) were

133 characteristic of ER-targeted proteins (>0.4): AT5g54170: 0.58, AT1G22890/STMP2: 0.63, 
AT2G45570.1/CYP76C2: 0.90, AT1G65500/STMP6: 0.63, AT1G33720/CYP76C6: 0.81,

AT3G04210.1/TN13: 0.50. We then assessed protein localization by confocal microscopy of Cterminal GFP fusions expressed under dexamethasone (Dex) control in Nicotiana benthamiana. Three candidates encoded by At5g54170, At1g65500/STMP6 and At2g45570, respectively, showed GFP signals that co-localized to varying extents with plastid signals (Figure 1C, Left panel). The subcellular locations of the At5g54170-encoded protein and STMP6 were further corroborated by fractionation and immunoblotting (Figure 1C, Right panel). This shows that the PATS algorithm can identify some plastid proteins besides AZI1 and EARLI1 from Arabidopsis.

\section{In silico analysis of subcellular targeting of the Arabidopsis HyPRPs}

PATS also predicted that seven HyPRP members, besides AZI1 and close paralogs, are plastid localized (Figure 1D). Arabidopsis HyPRPs are present in gene clusters in the genome (Supplementary Figure S1B). Some members of the HyPRPs family were previously named (AZI1, EARLI1, AZI3-AZI7, DEG27, DRN1, DHyPRP1, AIR1, AIR1B, ELP and CWLP); we

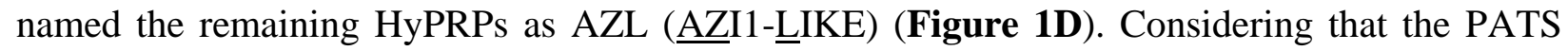
algorithm was trained using apicomplexa proteins, we also implemented another approach to identify AZI1-like plastid-targeting signals in Arabidopsis HyPRPs. TargetP and SignalP can efficiently recognize SP-like sequences and their cleavage sites in plant proteins. Therefore, we manually removed the N-terminal SP-like sequences identified by these algorithms. These SPlike-minus sequences of HyPRPs were then reanalyzed by TargetP and ChloroP (referred to herein as iterative TargetP, iTargetP or iChloroP) customized for plants with default cutoffs. By using SP-like-depleted sequences, the algorithms can analyze the N-terminal transit peptide-like 
157 sequences (called cTPs) for targeting specificity. About $70 \%$ of the Arabidopsis HyPRPs were

158 predicted to localize to plastids (Figure 1D, Supplementary Table S1). Moreover, the PATS

159 output had a high overlap with that of iTargetP/iChloroP, suggesting that apicoplast or iterative

160 chloroplast predictors can be successfully deployed to find candidates of plant plastid membrane

161 proteins with SP-like motifs.

162

163

164

165

166

167

168

169

170

171

172

173

174 nuclei (Supplementary Figure S1C). This raised the possibility that DRN1-GFP was

175 proteolyzed. Indeed, GFP was cleaved from a large pool of the protein (Supplementary Figure

176 S1C). Two previous mass spectrometry experiments assigned DRN1 to plastid membranes

177 (Peltier et al., 2004; Tomizioli et al., 2014), providing additional confidence that the protein is

178 plastid-associated. 

signal around them. In one case (AZL3), punctate GFP signals around many chloroplasts in

181 addition to PM signals were observed. While AZL6 showed similar localization to AZL3, the 182 chloroplast-associated punctate signals were less prominent. Finally, DHYPRP1 showed ER, $183 \mathrm{PM} / \mathrm{PD}$ and incomplete chloroplast rings (Figure 2A). The patterns showing ER, PM/PD and 184 chloroplast localization of HyPRPs were largely similar to the family member AZI1 and close 185 paralogs (Cecchini et al., 2015, 2020).

Evaluation by immunoblotting of chloroplast fractions from $N$. benthamiana leaves transiently expressing many HyPRP-GFP fusion proteins largely corroborated the microscopy (Figure 2B). We were unsuccessful in using immunoblotting to detect AZL3-GFP after

189 fractionation due to interference from the rubisco large subunit. Instead, we used a proteomics 190 approach and found AZL3 to be differentially enriched in chloroplast membranes of a 191 constitutively defense active Arabidopsis line (ACD6-1HA, (Lu et al., 2005)) relative to WT 192 (Figure 2C). Prior proteomics studies detected AZL3 in the thylakoid and envelope fractions, 193 respectively (Tomizioli et al., 2014; Kleffmann et al., 2004).

194 We next analyzed the localization patterns of Dex-inducible GFP fusions of the HyPRPs 195 AZL3, AZL13, AZL14 and ELP in stable Arabidopsis transgenic lines. Each transgene was 196 expressed in the respective mutant background. A portion of the GFP signal of these four fusion 197 proteins colocalized with chloroplast autofluorescence (Figure 3). Interestingly, while the GFP 198 signals for AZL13, AZL14 and ELP each showed a ring-like pattern surrounding the 199 chloroplasts, AZL3 had a more diffuse signal. This was further confirmed by analyzing the 200 fluorescence intensity profiles of the merged images (Figure 3, right most panel) and is 201 consistent with its import and association with thylakoid membranes (Tomizioli et al., 2014). 
Inspection of the features of the PRR for AZL3 compared with other HyPRPs that show the ring-

203 like pattern revealed that the AZL3 PRR lacks a KP motif present in others and also possesses a

204 unique repeated sequence (Supplementary Table S1). These and/or other differences in the

205 PRR composition (e.g. abundance of prolines relative to charged amino acid residues) may

206 account for differences in the suborganellar plastid localization patterns. Altogether, our data

207 show that for several HyPRPs, a pool of these proteins localizes to chloroplasts, as predicted.

\section{Several chloroplast-localized HyPRPs are associated with OEMs}

210 Except for AZL1 and AZL9, N-terminal regions of all HyPRPs have hydrophobicity

211 scores $>0.4$ on the Wimley-White scale (Supplementary Table S1). Thus, they do not have

212 features of classical signal-anchored OEM plastid proteins (Lee et al., 2011). Moreover, AZL1

213 and AZL9 (TMD hydrophobicity < 0.4; plastidial score) did not target to the chloroplasts as

214 indicated by confocal microscopy (Figure 2A). This suggests that HyPRPs follow a non-

215 canonical mechanism of targeting to chloroplast membranes, similar to the family member AZI1

216 (Cecchini et al., 2020).

217 To study the above possibility, we analyzed the chloroplast membrane association of

218 HyPRPs that showed ring-like localization around chloroplasts. We isolated chloroplasts from $N$.

219 benthamiana leaves transiently expressing GFP-tagged AZL2, AZL13, AZL14 and ELP. The

220 chloroplasts were then partitioned into pellet (membrane) and soluble fractions. Interestingly, all

221 these GFP-tagged HyPRPs were found to be associated with pellets (chloroplast membranes) as

222 evaluated by immunoblotting (Figure 4). Furthermore, intact chloroplasts from plants transiently

223 producing HyPRP-GFP fusion proteins treated with thermolysin resulted in HyPRP-GFP

224 proteolysis, consistent with localization to the OEMs. In contrast, the intermembrane space- 
227 indicate that several chloroplast-targeted HyPRPs reside in OEMs and that their C-terminal

228 regions topologically face the cytosol.

\section{The proline-rich region is required for the chloroplast targeting of HyPRPs} deletion of PRRs prevented all these HyPRP-GFP fusion proteins from targeting to chloroplasts,

240 consistent with a role for the PRRs in promoting the association of some Arabidopsis HyPRPs 241 with chloroplasts (Figure 5A-C).

244 domain is similarly dispensable for chloroplast targeting of a more distantly related HyPRP, we

245 generated ELPALTP-GFP. This truncated version of ELP associated with chloroplasts (Figure

246 5D). These data show that the PRRs play key roles in chloroplast targeting and are consistent

247 with the N-terminal region of HyPRPs being sufficient to confer this targeting. 


\section{Expression of $\mathrm{HyPRPs}$ is regulated in response to microbes}

The HyPRPs AZI1, EARLI1 and DRN1 are required for defense against pathogens and

252 of the literature and public data indicated that many $H y P R P$ transcripts are differentially

253 regulated by pathogens or pathogen-derived molecules (e.g. the PAMP flg22), suggesting that

254 they may play a role during plant defense responses (Table 1). Moreover, the expression of 255 many HyPRPs was also responsive to inoculation of roots with the beneficial microbe $P$. simiae 256 WCS417 (Stringlis et al., 2018). Analysis of publicly available gene expression data also 257 revealed that a number of HyPRPs are co-regulated to different degrees, suggesting they may be 258 involved in the same processes (Figure 6). The HyPRPs with the highest co-regulation values 259 were encoded by genes with strong chromosomal linkage (Supplementary Figure S1B).

We analyzed the transcript levels of several HyPRPs in WT after infection with a 261 pathogen capable of inducing strong local and systemic defenses, Pseudomonas cannabina pv.

262 alisalensis carrying AvrRpt2 (avirulent strain PmaDG6). Most $H y P R P$ transcripts assayed were 263 downregulated by $18 \mathrm{~h}$ post infection (Figure 6B). Because AZL3 showed increased protein

264 levels in the plastid membrane fraction of the constitutively defense-active line ACD6-1HA 265 (Figure 2C), we also assayed its transcript levels in these plants. Interestingly, the AZL3 266 transcript level was reduced in the ACD6-1HA line relative to WT (Figure 6C). Thus, AZL3 267 may be subject to post transcriptional regulation.

268 Together, previously published and our data show that the HyPRPs are differentially 269 regulated during biotic interactions (or simulated infection conditions provided by the ACD6270 1HA line) and thus may play a role, positive or negative, during interactions with microbes. 


\section{Several HyPRPs regulate SAR in Arabidopsis}

Since AZI1 and EARLI1 are required for systemic immunity, other HyPRPs with

274 chloroplast-associated pools might be similarly required. To test this, we evaluated local foliar

275 pathogen resistance and SAR using azl3, azl13, azl14 and elp T-DNA mutants. We used an anti-

276 sense line AZL2-AS (Jülke and Ludwig-Müller, 2015) for SAR experiments, since no azl2 mutant

277 was available.

In all mutants tested, the local (basal) resistance against a SAR-inducing (avirulent) as

279 well as a virulent Pseudomonas strain (PmaDG6 and PmaDG3, SAR-inducing and virulent

280 Pseudomonas cannabina pv. alisalensis, respectively) was similar to WT (Supplementary

281 Figure S3). In contrast, the azl3 and azll3 mutants and the AZL2-AS plants were SAR-deficient,

282 whereas the azll4 mutant still showed robust SAR, similar to WT (Figure 7). SAR response gain

283 values (Jiang et al., 2021) of the different genotypes supported this conclusion (Figure 7B, D).

284 Interestingly, the elp mutant showed significantly higher resistance levels to PmaDG3 in

285 systemic leaves during SAR and an increased response gain compared to the WT (Figure 7A,

286 B). This unusual "super-SAR" phenotype was confirmed in elp-2, another T-DNA allele

287 (Supplementary Figure S4A).

288 We also analyzed the induction of SAR marker PR1 in the uninfected systemic leaves of

289 WT, azl3, azll3, azll4, and elp mutants after local immunization with PmaDG6 or mock

290 treatment. Consistent with the observed bacterial growth (Figure 7A), there was increased

291 accumulation of PR1 in systemic leaves of elp compared to WT, 72 hours post local PmaDG6

292 infection (Figure 7E, F). Moreover, azl3 and azll3 mutants showed significantly lower PR1 than

293 WT in systemic leaves at both 48 and 72 hpi (Figure 7E, F). 
We performed complementation experiments (Figure 7G) using the transgenic plants in

295 which GFP fusions were studied in Figure 3. The expression of HyPRPs was induced by

296 spraying whole plants with Dex plus $0.04 \%$ Tween $20,21 \mathrm{~h}$ before infection. In addition to

297 visualizing GFP (Figure 3), transgene expression was confirmed by RT-PCR (Figure 7H).

298 Dex:AZL3\#1 and Dex:AZL13\#1 transgenic lines were complemented for SAR and were

299 indistinguishable from WT plants (Figure 7G, I). Independent Dex:AZL3\#2 and Dex:AZL13\#2

300 lines were also complemented for SAR (Supplementary Figure S4 B, C). The Dex:ELP\#1 line

301 was completely SAR-deficient, indicating that the dosage of ELP (overexpression) strongly

302 affects SAR (Figure 7G, I). The contrasting phenotypes of the mutant (super-SAR) and

303 Dex:ELP\#1 line (SAR-deficient) strongly position this particular HyPRP as a negative regulator

304 of SAR.

305

\section{HyPRPs are required for ISR}

Many HyPRPs are differentially expressed in roots, particularly during the interaction of

308 roots with $P$. simiae WCS417 (Table 1; (Stringlis et al., 2018)). Therefore, we tested the role of

309 several HyPRPs with plastid-associated pools in ISR. As shown in Figure 8, azl3, azll3 and

310 azll4 mutants were unable to mount ISR. In contrast, only WT and elp showed significant (and

311 similar) ISR (Figure 8A, B). These data indicate that several newly characterized HyPRPs have

312 positive roles in promoting ISR.

314 HyPRPs determine colonization, developmental and/or growth-promoting effects of root-

\section{5 associated bacteria}


316 Strain $P$. simiae WCS417, used to stimulate ISR, also promotes plant growth and lateral root

317 formation in Arabidopsis (Haney et al., 2015; Pieterse et al., 2014). Unlike WT, upon treatment

318 of roots with $P$. simiae WCS417, the azll3 and azll4 mutants showed no significant increase in

319 biomass or lateral root numbers. The azl3 mutant also failed to show a biomass increase, but did

320 display increased lateral root numbers (even higher than WT, Figure 9). As was seen with the

321 SAR assay, the elp mutant was hyper-responsive, showing both biomass and lateral root numbers

322 that exceeded that seen in WT upon P. simiae WCS417 inoculation.

We noticed that azll4 and to a lesser extent azl3 and azll3 had shorter primary root

324 lengths than WT in the absence of bacteria (Figure 9D). However, azl3 and azll3 showed

325 similar increased primary root growth as WT in response to WCS417. In contrast, the elp mutant

326 failed to show increased primary root growth and azll4 showed reduced growth in response to $P$.

327 simiae WCS417.

colonization (Haney et al., 2015). Therefore, we analyzed the root bacteria attachment in WT and

mutant plants after growing the different genotypes hydroponically in 48-well plates on a Teflon

mesh (Figure 9E). Seedlings were initially grown with $2 \%$ sucrose to avoid differences in root

332 development between genotypes and thus allowing subsequent comparisons of colonization of

333 roots in media without sucrose (see Methods). Interestingly, although there was no significant

334 difference in the bacterial growth (thought to be stimulated by root exudation) in the well media

335 in which plants were growing, azl3, azl13 and azll4 mutant roots supported less P. simiae

336 colonization compared to WT or elp mutant plants (Figure 9F).

Altogether, these data suggest that rhizosphere-mediated differences in $P$. simiae-induced

338 responses may partly depend on the host's ability to support bacterial growth on the roots. 
Importantly, these results suggest that HyPRPs have varied positive and negative role(s) in the

340 roots including supporting beneficial microbe colonization and impacting root architecture and

341 growth promotion.

\section{Discussion}

345 Most HyPRP family proteins have SP-like/HD domains at their $\mathrm{N}$ termini followed by varying

346 lengths of unstructured PRRs. We previously proposed that the N-terminal bipartite signal

347 formed by SP-like/HD (TMD) + PRR define the subcellular targeting of AZI1 and paralogs to

348 plastids (Cecchini et al., 2015, 2020). By using algorithms (PATS, iTargetP/iChloroP) that

349 recognize the N-terminal signal (SP-like/TMD + PRR/cTP or just PRR/cTP), we predicted with

350 about $80 \%$ success the subcellular targeting of many members of the HyPRP family in

351 Arabidopsis. Predictions that were not correct largely correlated with HyPRPs harboring shorter

352 PRRs. Importantly, the PATS algorithm also identified plausible bipartite signals in a large

353 number of Arabidopsis proteins. PATs correctly predicted $\geq 33 \%$ of the non-HyPRP proteins that

354 we tested. The non-HyPRP plastid protein STMP6 is among a group of proteins recently

355 proposed to be secreted (Yu et al., 2020). Many proteins in this group, regulated in response to

356 pathogens, are PATS-positive and are thus also good candidates for having a pool that localizes

357 to plastids.

358 Plastid targeting signals are diverse, consisting of multiple sequence motifs that are

359 distinct or conserved (Bruce, 2001; Lee et al., 2008). Of note is that Pro residues in cTPs mediate

360 efficient translocation of TMD-containing proteins through the chloroplast envelopes (Lee et al.,

361 2018). A Ser/Pro-rich domain targets or facilitate targeting/anchoring of other inner envelope 
362 proteins (Küchler et al., 2002; Tripp et al., 2007). Longer PRRs together with the TMDs may

363 similarly help in chloroplast membrane-anchoring of HyPRPs (Cecchini et al., 2020). Charged

364 amino acids (intrinsic charge or those caused by post translational modifications) may play a role

365 in targeting, given their prevalence in PRRs (Supplementary Table S1). Considering that AZI1

366 has a non-cleavable N-terminal signal sequence, these domains in other HyPRPs also are

367 unlikely to be cleaved during their import (Cecchini et al., 2020). This is consistent with the

368 finding that most known proteins in the outer envelope lack a cleavable targeting signal

369 (Hofmann and Theg, 2005). Thus, it is plausible that other plastid-membrane targeted HyPRPs

370 follow the targeting mechanism similar to AZI1, with SP-like/TMD + PRR guiding their plastid

371 association.

372 We showed that four HyPRPs (AZL2, AZL13, AZL14 and ELP) are OEM plastid

373 proteins. Two HyPRPs (AZL3 and DRN1) were previously suggested to be thylakoid and/or

374 envelope proteins, as evidenced by their presence in proteomics datasets (Peltier et al., 2004;

375 Tomizioli et al., 2014; Kleffmann et al., 2004). While thylakoid samples could be contaminated

376 with plastid envelope proteins, our microscopy of AZL3 in Arabidopsis is consistent with a

377 thylakoid location. This suggests that bipartite signals, depending on their composition, may

378 permit the targeting of proteins to plastid envelopes or the internal plastid thylakoid membrane.

379 Figure $\mathbf{1 0}$ summarizes the localization patterns of HyPRPs, and by analogy to

380 AZI1/EARLI1, proposes that they are needed for mobilizing signals. The multiple roles of

381 HyPRPs in microbial responses that were found previously (Cecchini et al., 2015, 2019b; Dhar et

382 al., 2020) and herein are shown in Figure 10B. ELP is unique among the HyPRPs in how it

383 impacts responses to microbes, as it is a negative regulator of both SAR and growth/development

384 responses to the root-colonizing microbe WCS417. The elp mutant shows increased response 
gain and PR1 accumulation during SAR, but does not affect basal resistance, suggesting increased signaling output to distal tissue. This mutant also has normal root colonization, but has increased plant growth and lateral root numbers relative to WT, further supporting enhanced signaling. However, ISR is not affected in the elp mutant, indicating distinct requirements for the developmental responses and ISR. This is consistent with certain ISR-defective mutants showing robust growth and developmental responses when co-cultivated with WCS417 (Zamioudis et al., 2013).

Some mutants in this study (azl3 and azl13) are affected in both SAR and ISR, similar to

393 the azil and earlil mutants of HyPRPs (Jung et al., 2009; Cecchini et al., 2015). For the SAR

394 phenotypes, the defects are linked to long distance signaling, since the growth of the immunizing

395 pathogen is normal. However, the $a z l 3$, azll3 and azll4 mutants show possible local signaling defects evidenced by lower colonization of roots with WCS417 relative to that seen in WT. It is possible that other root-related defects in these mutants arise from low root colonization. A certain threshold of rhizobacterial colonization may be needed to induce the downstream root development, plant growth and ISR phenotypes. However, azl3 still shows lateral root induction,

400 indicating that there is enough colonization to cause signaling for this phenotypic output. Lateral 401 root induction in azl3 may occur due to the presence of other HyPRPs (AZL13, AZL14, AZI1 402 and/or EARLI1 (Cecchini et al., 2019b)). This would implicate other HyPRPs in regulating both 403 colonization as well as the downstream signaling for lateral root induction.

405 root number increases) require different thresholds of bacterial colonization of the roots.

406 Alternatively, all outputs may require the same threshold, but the certain HyPRPs may be 407 differentially needed for multiple steps: colonization and downstream signaling to cause growth, 
408 ISR and/or lateral root production. A genome-wide association mapping study (Wintermans et

409 al., 2016) identified different markers that were independently associated with shoot growth

410 promotion, lateral root formation and primary root length changes in response to WCS417. This

411 finding supports the idea that there are separate pathways (or branches of a pathway) that

412 independently regulate the different phenotypic outputs. Interestingly, two HyPRPs (DEG27,

413 AZL2) are within 5-10 KB of a marker associated with regulating the lateral root changes.

414 Despite having positive roles in regulating interactions with/responses to microbes,

415 transcript levels of many HyPRPs are down regulated by microbe-related signals. This could

416 reflect negative feedback to modulate signaling. However, in plants with constitutive defenses

417 (ACD6-1HA) the transcript level of AZL3 is low, while the plastid membrane-associated protein

418 level is high relative to WT. This suggests that there is complexity to the regulation of HyPRPs.

419 Some HyPRPs may become stabilized by post translational modification, since they have kinase

420 motifs (SP/TP, Supplementary Table S1). AZI1's PRR is a kinase substrate in vitro (Pitzschke

421 et al., 2014).

422 HyPRPs are similar and they locate to several types of membranes. This could permit the

423 formation of membrane contact sites and complexes with other HyPRPs/LTPs in different

424 places. Multiple contact sites between plastids, ER and PM, comprising of LTPs, are the sites of

425 exchange of small molecule signals (Andersson et al., 2007; Li et al., 2020; Toulmay and Prinz,

426 2011). HyPRPs are likely enriched in these sites for the mobilization/transport of immune and/or

427 developmental and growth signals. Supporting this, AZI1 and EARLI1 can interact and mobilize

428 the SAR signal AZA, which also can trigger AZI1-/EARLI1-dependent root architecture changes

429 (Cecchini et al., 2019b, 2015). Moreover, because AZI1/EARLI1 physically interact with non-

430 HyPRP LTP superfamily member (i.e. DIR1) in membrane contact sites to modulate systemic 
431 responses (Cecchini et al., 2015; Yu et al., 2013), it is also possible that other positive regulatory

432 HyPRPs similarly form active homo or hetero-oligomeric LTP complexes at the key

433 sites/structures for transmission of developmental, growth and/or defense signals to regulate

434 systemic defense responses. The presence of so many HyPRPs may allow the movement of

435 signals at different rates (to reach specific thresholds) or enable movement of various signals that

436 require different membrane-paths to move. For example, to reach the xylem to move signals

437 aboveground may require the use of different contact sites compared to signals reaching

438 parenchyma for lateral root stimulation. Thus, different signals (or the amount of signal moved)

439 could affect and explain different specific phenotypes.

\section{Methods}

\section{Plants, bacteria and plasmids}

444 All the Arabidopsis thaliana plants used were Columbia-0 (Col-0) ecotype. The plants used for 445 infection were grown in soil (Berger, BM1:BM2; 50:50 mix) or Jiffy- $7^{\circledR}$ peat pellets (for ISR, 446 Hummert International, \# 14-23700) at 12h day/night cycle as previously described (Cecchini et 447 al., 2015). The following Arabidopsis accessions were obtained from ABRC: At1g12090 (elp, 448 SALK_147582C; elp-2, SAIL_532_C09), At2g10940 (azl3, SALK_083118C), At5g46890 449 (azl13, SALK_065132), At5g46900 (azl14, SALK_096750C). At1g62510 AZL2-AS line was 450 described previously (Jülke and Ludwig-Müller, 2015). The transgenic line ACD6-1HA (Lu et 451 al., 2005) used for proteomic analysis was grown at 16h day/8h night cycle. pt-gk (cTP-GFP) 452 transgenic line from ABRC (CS16266) was used as a chloroplast marker. Arabidopsis HyPRP 453 complementation lines were generated by floral dip method using Agrobacterium tumefaciens 
454 C58C1 suspensions (Clough and Bent, 1998). N. benthamiana used for localization studies were

455 grown in soil at $24^{\circ} \mathrm{C}$ under $16 \mathrm{~h}$ light/8h night regime. For $A$. tumefaciens-mediated transient

456 assays, 4-5-week-old $N$. benthamiana plants were used.

457 Bacterial strains used for infections or colonization were virulent Pseudomonas

458 cannabina $p v$. alisalensis (formerly Pseudomonas syringae pv. maculicola strain ES4326)

459 carrying an empty vector (PmaDG3), the avirulent isogenic strain carrying avrRpt2 (PmaDG6)

460 and Pseudomonas simiae WCS417 (formerly Pseudomonas fluorescens WCS417r).

461 The full-length coding sequences or the truncated versions of HyPRPs were amplified by

462 PCR and cloned by Gateway procedure into Dexamethasone (Dex)-inducible plant expression

463 vectors pBAV150 (C-terminal GFP tag) or pBAV154 (C-terminal HA tag) (Cecchini et al.,

464 2015). Full-length coding sequences of HyPRPs in pBAV150 vector were used to transform

465 Arabidopsis to generate complementation lines. To generate $\triangle \mathrm{PRR}$ variants, proper fragments

466 were generated with overlapping primers and linked by PCR. $\triangle$ LTP variants were generated

467 using non-overlapping primers in PCR. All the primers and constructs used in this study are

468 listed in (Supplementary Table S2).

$470 \quad$ In silico analysis of subcellular targeting

471 For bipartite plastid targeting signal prediction, we analyzed the full-length sequences of 472 Arabidopsis proteome (ATpepTAIR10) by using PATS software

473 (http://modlabcadd.ethz.ch/software/pats/) or an iterative method (iTargetP/iChloroP). In the 474 iterative method, N-terminal SP-like regions as determined by TargetP 475 (http://www.cbs.dtu.dk/services/TargetP-1.1/) or SignalP

476 (http://www.cbs.dtu.dk/services/SignalP/), varying between 22 and 27 amino acids (except for 
477 AZI7 with 30 amino acids as SP-like) were removed in silico and the remaining sequences were

478 analyzed by TargetP/ChloroP to determine the presence of chloroplast transit peptide following

479 the SP-like region.

480 The GO analysis was done as described (Bonnot et al., 2019). The list of representative

481 enriched GO terms was obtained using Panther

482 (https://www.arabidopsis.org/tools/go_term_enrichment.jsp) and REVIGO (http://revigo.irb.hr)

483 tools.

484

485 Confocal Imaging

486 9-12 day-old Arabidopsis seedlings or 4-week old $N$. benthamiana leaves expressing the

487 different HyPRP versions fused at C-terminal with GFP were visualized by confocal microscopy.

488 Zeiss LSM710 and LSM800 laser scanning confocal microscopes were used to visualize GFP

489 fluorescence (excitation $=488 \mathrm{~nm}$, emission $=509 \mathrm{~nm}$ ) and chlorophyll autofluorescence

490 (excitation $=631 \mathrm{~nm}$, emission $=647 \mathrm{~nm})$. LD C-Apochromat 40x/1.1 W Korr $($ LSM710) and

491 EC Plan-Neofluor 40x/1.3 (LSM800) objectives were used for taking images. The images are

492 optical sections captured at 1024 x 1024 pixels scanning resolution in maximum speed mode.

493 Fluorescence of GFP and chlorophyll was acquired in sequential acquisition mode. Plant tissues

494 were mounted in perfluorodecalin (Strem Chemicals, Inc.) for optical enhancement. Images were

495 processed by ImageJ (Fiji) and Adobe photoshop software.

496

497 Fractionation

498 N. benthamiana leaves, transiently transformed with A. tumefaciens harboring different 499 constructs, were used to isolate chloroplasts as described in (Cecchini et al., 2015) with minor 
modifications. Briefly, leaves (0.5-1g) were homogenized in XpI buffer (0.33 M sorbitol, $50 \mathrm{mM}$

501 HEPES pH 7.5, $2 \mathrm{mM}$ EDTA, $1 \mathrm{mM} \mathrm{MgCl}_{2}, 0.25 \%$ BSA, 0.1\% sodium ascorbate and protease

502 inhibitors) using polytron (Kinematica) and filtered through two layers of Miracloth

503 (Calbiochem, \# 475855). The filtrate was centrifuged at $5000 \mathrm{rpm}$ for 5 minutes and the pellet

504 was resuspended in $0.4 \mathrm{ml}$ of $\mathrm{XpI}$ buffer. The resuspended pellet was loaded on Percoll (GE, \#

505 17089101) gradient (80\% and 40\%) and centrifuged at $13,000 \mathrm{rpm}$ to isolate the intact

506 chloroplasts. The recovered intact chloroplasts at the interphase were resuspended in $0.5 \mathrm{ml} \mathrm{XpI}$

507 buffer and centrifuged at $3000 \mathrm{rpm}$ for 5 minutes to remove the Percoll impurities. The purity of

508 the chloroplast fractions was checked using specific markers by immunoblotting.

509 To obtain enriched membrane and soluble fractions, the intact chloroplasts were partitioned

510 into membrane (pellet) and soluble fractions by centrifugation. To do this, the plastids were

511 resuspended in protease free ice-cold water containing protease inhibitor cocktail (Thermo

512 Scientific, \#A32965). For complete lysis, the chloroplasts were incubated on ice for 45-60 min

513 followed by incubation at $-80^{\circ} \mathrm{C} \mathrm{O} / \mathrm{N}$. The samples were then thawed on ice and centrifuged at

$51413,000 \mathrm{rpm}$. The soluble fraction was recovered, and the pellet was resuspended in $1 \mathrm{XPBS}(+1 \%$

515 SDS and protease inhibitor cocktail).

\section{Thermolysin assay}

518 Proteolysis by thermolysin was performed as described in (Cecchini et al., 2015). Briefly, intact

519 chloroplast fractions were resuspended in $1 \mathrm{X}$ import buffer (0.33 M sorbitol, $50 \mathrm{mM} \mathrm{HEPES,} \mathrm{pH}$

520 8.0) and treated with thermolysin (to a final concentration of $0.1 \mathrm{mg} / \mathrm{ml}$ ). The reaction was

521 quenched by adding 10mM EDTA. The chloroplasts were carefully separated on $40 \%$ Percoll

522 cushion. 


\section{Immunoblotting}

525 Equal amounts of total proteins were separated on 12\% SDS-PAGE and transferred to PVDF 526 membranes (Millipore, \#IPVH00010). The concentration of proteins was determined by 527 Bradford assay. The following primary antibodies were used in this study: PR1 antibody 528 (Agrisera, \#AS10687; 1:2,500), GFP monoclonal antibody (Takara, \# 632375; 1:2,000), Bip2 529 antibody (Agrisera, \#AS09481; 1:4,000), Tic110 antibody (a gift from Masato Nakai; 1:3,000), 530 chloroplast Hsp70 (a gift from Thomas Leustek; 1:12,000), Toc75 (Agrisera, \#AS08351, 531 1:3000). Horseradish peroxidase-conjugated anti-mouse (Invitrogen, \#SA1-100; 1:4,000), anti532 guinea pig (Sigma, \#A5545; 1:100,000), or anti-rabbit (Thermo Scientific, \#32460: 1:5,00) 533 secondary antibodies were used. The bands were detected by using SuperSignal pico/femto 534 chemiluminescence kits (Thermo Scientific; \#'s1859022/3 and 1859674/5).

\section{Transcript analysis}

537 Total RNA was isolated with the RNeasy Plant Mini Kit (Qiagen) and cDNA was synthesized 538 using was the Reverse Transcriptase SuperScript III and Oligo (dT) $)_{20}$ primers (ThermoScientific) 539 according to the manufacturer's instructions. HyPRP expression (in WT/ACD6-1HA) was 540 analyzed by quantitative RT-PCR using SYBR Premix Ex Taq (Takara) in a Bio-Rad Real-Time

541 CFX96TM C1000 system (Bio-Rad) according to the manufacturer's instructions. For Dex:AZL

542 lines, relative transcript levels were checked by RT-PCR (optimized for cycle \#s). EF1 $\alpha$ was 543 used as a reference for normalization. 
546 The evaluation of bacterial pathogen growth was caried as described (Jung et al., 2009). Briefly,

547 26-28 day-old wild-type and HyPRP mutant plants were syringe-infiltrated with PmaDG3 or

548 PmaDG6 $\left(\mathrm{OD}_{600}=0.0001\right)$. Growth was quantified using eight equal sized leaf discs from

549 different plants 3 days after bacterial inoculation. All graphs of colony forming units (cfus) were

550 plotted as $\log _{10}$ values.

551 For SAR assays (Jiang et al., 2021), local leaves of 26-28-day old plants were syringe-infiltrated 552 with mock $\left(10 \mathrm{mM} \mathrm{MgSO}_{4}\right)$ or PmaDG6/avrRpt2 $\left(\mathrm{OD}_{600}=0.01\right)$. Two days after the primary 553 infection, systemic leaves were infiltrated with PmaDG3 $\left(\mathrm{OD}_{600}=0.0001\right)$. The local leaves were 554 removed from the plants prior to the secondary challenge and the plants were covered with 555 plastic domes following the secondary challenge. Three days post-secondary challenge, equal 556 sized leaf discs were collected and ground in $10 \mathrm{mM} \mathrm{MgSO}_{4}$. The ISR assay was performed 557 exactly as described (Cecchini et al., 2019a) with PmaDG3 OD $_{600}$ of 0.0001-0.0002. The 558 response gain of SAR or ISR was calculated as described (Jiang et al., 2021).

Plant growth promotion assays of whole plants and roots

561 Arabidopsis were grown vertically at $\sim 20^{\circ} \mathrm{C}$ with $16 \mathrm{~h}$ day/8h night on small square plates 562 containing MS media (GibcoBRL, Life Technologies) without sugar as previously described 563 (Haney et al., 2015). Briefly, ten seeds were germinated on plates and five days after plating, 564 seedlings were thinned to five seedlings per plate and roots inoculated with $1 \mu \mathrm{l} 10 \mathrm{mM} \mathrm{MgSO}_{4}$ 565 (Mock) or Pseudomonas simiae WCS417 (OD $600=0.001)$. After 10 days, scanned (Epson) plant 566 images were used to count lateral roots and measure primary root lengths. Subsequently the fresh 567 weights were determined. 


\section{Root colonization assay}

570 Root colonization assay was performed in 48-well plates as described (Haney et al., 2015). 250 $\mu 1$

571 MS media (Sigma, \#M5519-10L) containing 2\% sucrose was added to the wells of 48-well tissue

572 cultures plates (BD Falcon). Round Teflon mesh disks (McMaster- Carr \#1100t41) were

573 sterilized by autoclaving and placed into the wells. A single surface sterilized and imbibed seed

574 was placed at the center of each disc. Seedlings were subsequently grown at $\sim 20^{\circ} \mathrm{C}$ with $16 \mathrm{~h}$

575 day/8 h dark for 10 days. The MS media was replaced with $270 \mu$ of fresh MS media without

576 sugar. Two days after the media change, wells were inoculated with $30 \mu$ (final $\mathrm{OD}_{600}=$

577 0.00002) of $P$. simiae WCS417 suspended in water. After 7 days, seedlings were removed, and

578 roots and well media analyzed for bacterial amount (six seedlings were combined per data point).

579 The root extracts prepared by grinding and the corresponding well media were plated by serial

580 dilution to determine cfus.

\section{$581 \quad$ LC-MS/MS}

582 Approximately 15g Arabidopsis shoots of WT and ACD6-1HA were homogenized and the

583 chloroplasts separated on Percoll gradients ( $80 \%$ and $40 \%)$. The total plastid membrane fraction

584 obtained after centrifugation was used for LC-MS/MS. Total plastid membrane proteins were

585 analyzed by Q-Exactive Orbitrap mass spectrometer. Relative quantification was performed

586 using Maxquant software. The raw data was searched against the Arabidopsis thaliana database,

587 as well as reversed protein sequences, and common contaminants. The search results were

588 filtered to be within $1 \%$ false discovery rate and label-free quantification using normalized

589 protein abundances was performed to assess differential protein expression between WT and the

590 transgenic expressing ACD6-1HA. 


\section{Plotting and statistical analysis.}

593 The graphs were plotted in R studio (v.3.6.1/4.1) using ggplot2 package (v3.3.2) and all statistics

594 were done using agricolae package (v1.3.3).

\section{Acknowledgments}

This work was supported by NSF grant IOS-1456904 to J.T.G. Z.Z.B. was supported by a

600 fellowship from SERB/IUSSTF. At the University of Chicago, we thank Dr. Atif Khan for help

601 with R programming, Dr. Joanna Jelenska for experimental advice, Professor Stephen Kron and

602 Donald Wolfgeher for assistance with the proteomics experiment, Greenberg lab members for

603 reading and commenting on the manuscript and Dequantarius J. Speed for useful discussions.

604 We thank Professor Jutta Ludwig-Mueller, Technische Universität Dresden for providing the

605 AZL2-AS lines and Dr. Jennifer Morrell-Falvey at Oak Ridge National Laboratory for helpful

606 comments on the manuscript.

607

608 Author contributions

609 J.T.G., N.M.C. and Z.Z.B. designed the experiments. Z.Z.B, N.M.C, A.T.S., C.T.H., R.C.F., and

610 E.A. performed experiments. Z.Z.B, N.M.C., A.T.S. and J.T.G. performed bioinformatic analysis

611 or data mining. Z.Z.B, N.M.C and J.T.G. wrote the manuscript.

612 


\section{Supplemental data}

614 Supplementary Figure S1. Over-represented GO terms for biological processes in PATS plastid

615 predicted proteins, location of HyPRPs in Arabidopsis genome and GFP cleavage in DRN1.

616 Supplementary Figure S2. ClustalW alignment of Arabidopsis HyPRPs.

617 Supplementary Figure S3. Local disease resistance of HyPRP mutants against Pseudomonas

618 syringae pv. maculicola strains.

619 Supplementary Figure S4. SAR in elp-2 allelic mutant and Dex-inducible complementation

620 lines.

621 Supplementary Dataset S1. List of all PATS positive proteins of Arabidopsis.

622 Supplementary Table S1. CPRs, PRRs, WWH scores, experimental and predicted plastid

623 targeting of Arabidopsis HyPRPs.

624 Supplementary Table S2. List of primers and constructs used in this study.

References

Albert, I., Hua, C., Nürnberger, T., Pruitt, R.N., and Zhang, L. (2020). Surface Sensor Systems in Plant Immunity. Plant Physiol 182: 1582-1596.

Andersson, M.X., Goksör, M., and Sandelius, A.S. (2007). Membrane contact sites: physical attachment between chloroplasts and endoplasmic reticulum revealed by optical manipulation. Plant Signal Behav 2: 185-187.

Ascencio-Ibáñez, J.T., Sozzani, R., Lee, T.-J., Chu, T.-M., Wolfinger, R.D., Cella, R., and Hanley-Bowdoin, L. (2008). Global Analysis of Arabidopsis Gene Expression Uncovers a Complex Array of Changes Impacting Pathogen Response and Cell Cycle during Geminivirus Infection. Plant Physiol. 148: 436-454.

Baum, S., Reimer-Michalski, E.-M., Bolger, A., Mantai, A.J., Benes, V., Usadel, B., and Conrath, U. (2019). Isolation of Open Chromatin Identifies Regulators of Systemic Acquired Resistance. Plant Physiol. 181: 817-833. 
Beckers, G.J.M., Jaskiewicz, M., Liu, Y., Underwood, W.R., He, S.Y., Zhang, S., and Conrath, U. (2009). Mitogen-activated protein kinases 3 and 6 are required for full priming of stress responses in Arabidopsis thaliana. Plant Cell 21: 944-953.

Berendsen, R.L., Pieterse, C.M.J., and Bakker, P.A.H.M. (2012). The rhizosphere microbiome and plant health. Trends Plant Sci 17: 478-486.

Bobik, K. and Burch-Smith, T.M. (2015). Chloroplast signaling within, between and beyond cells. Front Plant Sci 6: 781.

Bonnot, T., Gillard, M., and Nagel, D. (2019). A Simple Protocol for Informative Visualization of Enriched Gene Ontology Terms. BIO-PROTOCOL 9.

Bruce, B.D. (2001). The paradox of plastid transit peptides: conservation of function despite divergence in primary structure. Biochimica et Biophysica Acta (BBA) - Molecular Cell Research 1541: 2-21.

Caplan, J.L., Kumar, A.S., Park, E., Padmanabhan, M.S., Hoban, K., Modla, S., Czymmek, K., and Dinesh-Kumar, S.P. (2015). Chloroplast Stromules Function during Innate Immunity. Dev Cell 34: 45-57.

659 Carella, P. (2020). Xylem-Mobile Oxylipins Are Critical Regulators of Induced Systemic Resistance in Maize. Plant Cell 32: 13-14.

Cecchini, N., Song, Y., Roychoudhry, S., Greenberg, J., and Haney, C. (2019a). An Improved Bioassay to Study Arabidopsis Induced Systemic Resistance (ISR) Against Bacterial Pathogens and Insect Pests. BIO-PROTOCOL 9.

Cecchini, N.M., Roychoudhry, S., Speed, D.J., Steffes, K., Tambe, A., Zodrow, K., Konstantinoff, K., Jung, H.W., Engle, N.L., Tschaplinski, T.J., and Greenberg, J.T. (2019b). Underground Azelaic Acid-Conferred Resistance to Pseudomonas syringae in Arabidopsis. MPMI 32: 86-94.

Cecchini, N.M., Speed, D.J., Roychoudhry, S., and Greenberg, J.T. (2020). Kinases and protein motifs required for AZI1 plastid localization and trafficking during plant defense induction. Plant J.

Cecchini, N.M., Steffes, K., Schläppi, M.R., Gifford, A.N., and Greenberg, J.T. (2015). Arabidopsis AZI1 family proteins mediate signal mobilization for systemic defence priming. Nat Commun 6: 7658.

Chen, Y.-L., Chen, L.-J., and Li, H.-M. (2016). Polypeptide Transport-Associated Domains of the Toc75 Channel Protein Are Located in the Intermembrane Space of Chloroplasts. Plant Physiol 172: 235-243. 
Clough, S.J. and Bent, A.F. (1998). Floral dip: a simplified method for Agrobacterium-mediated transformation of Arabidopsis thaliana. Plant J 16: 735-743.

Dempsey, D.A., Vlot, A.C., Wildermuth, M.C., and Klessig, D.F. (2011). Salicylic Acid biosynthesis and metabolism. Arabidopsis Book 9: e0156.

Dhar, N., Caruana, J., Erdem, I., and Raina, R. (2020). An Arabidopsis DISEASE RELATED NONSPECIFIC LIPID TRANSFER PROTEIN 1 is required for resistance against various phytopathogens and tolerance to salt stress. Gene: 144802.

Efthimiadou, A., Katsenios, N., Chanioti, S., Giannoglou, M., Djordjevic, N., and Katsaros, G. (2020). Effect of foliar and soil application of plant growth promoting bacteria on growth, physiology, yield and seed quality of maize under Mediterranean conditions. Sci Rep 10: 21060 .

Emanuelsson, O., Nielsen, H., Brunak, S., and von Heijne, G. (2000). Predicting Subcellular Localization of Proteins Based on their N-terminal Amino Acid Sequence. Journal of Molecular Biology 300: 1005-1016.

Emanuelsson, O., Nielsen, H., and Heijne, G.V. (1999). ChloroP, a neural network-based method for predicting chloroplast transit peptides and their cleavage sites. Protein Sci. 8: 978984.

Fu, Z.Q. and Dong, X. (2013). Systemic acquired resistance: turning local infection into global defense. Annu Rev Plant Biol 64: 839-863.

Gupta, A. and Senthil-Kumar, M. (2017). Transcriptome changes in Arabidopsis thaliana

Haney, C.H., Samuel, B.S., Bush, J., and Ausubel, F.M. (2015). Associations with rhizosphere infected with Pseudomonas syringae during drought recovery. Sci Rep 7: 9124.

Hartmann, M., Kim, D., Bernsdorff, F., Ajami-Rashidi, Z., Scholten, N., Schreiber, S., Zeier, T., Schuck, S., Reichel-Deland, V., and Zeier, J. (2017). Biochemical Principles and Functional Aspects of Pipecolic Acid Biosynthesis in Plant Immunity. Plant Physiol. 174: $124-153$.

Helle, S.C.J., Kanfer, G., Kolar, K., Lang, A., Michel, A.H., and Kornmann, B. (2013). Organization and function of membrane contact sites. Biochim Biophys Acta 1833: $2526-2541$.

Hofmann, N.R. and Theg, S.M. (2005). Chloroplast outer membrane protein targeting and insertion. Trends Plant Sci 10: 450-457.

Howard, B.E., Hu, Q., Babaoglu, A.C., Chandra, M., Borghi, M., Tan, X., He, L., WinterSederoff, H., Gassmann, W., Veronese, P., and Heber, S. (2013). High-throughput RNA sequencing of pseudomonas-infected Arabidopsis reveals hidden transcriptome complexity and novel splice variants. PLoS One 8: e74183. 
Hruz, T., Laule, O., Szabo, G., Wessendorp, F., Bleuler, S., Oertle, L., Widmayer, P., Gruissem, W., and Zimmermann, P. (2008). Genevestigator v3: a reference expression database for the meta-analysis of transcriptomes. Adv Bioinformatics 2008: 420747.

Jaskiewicz, M., Conrath, U., and Peterhänsel, C. (2011). Chromatin modification acts as a memory for systemic acquired resistance in the plant stress response. EMBO Rep 12: 5055 .

Jayasinghe, S., Hristova, K., and White, S.H. (2001). Energetics, stability, and prediction of transmembrane helices11Edited by G. von Heijne. Journal of Molecular Biology 312: 927-934.

Jiang, S.-C., Engle, N.L., Banday, Z.Z., Cecchini, N.M., Jung, H.W., Tschaplinski, T.J., and Greenberg, J.T. (2021). ALD1 accumulation in Arabidopsis epidermal plastids confers local and nonautonomous disease resistance. J Exp Bot.

Jülke, S. and Ludwig-Müller, J. (2015). Response of Arabidopsis thaliana Roots with Altered Lipid Transfer Protein (LTP) Gene Expression to the Clubroot Disease and Salt Stress. Plants 5: 2.

Jung, H.W., Tschaplinski, T.J., Wang, L., Glazebrook, J., and Greenberg, J.T. (2009). Priming in Systemic Plant Immunity. Science 324: 89-91.

Kachroo, A. and Kachroo, P. (2020). Mobile signals in systemic acquired resistance. Curr Opin Plant Biol 58: 41-47.

Kleffmann, T., Russenberger, D., von Zychlinski, A., Christopher, W., Sjölander, K., Gruissem, W., and Baginsky, S. (2004). The Arabidopsis thaliana Chloroplast Proteome Reveals Pathway Abundance and Novel Protein Functions. Current Biology 14: 354-362.

Küchler, M., Decker, S., Hörmann, F., Soll, J., and Heins, L. (2002). Protein import into chloroplasts involves redox-regulated proteins. EMBO J 21: 6136-6145.

Lee, D.W., Kim, J.K., Lee, S., Choi, S., Kim, S., and Hwang, I. (2008). Arabidopsis NuclearEncoded Plastid Transit Peptides Contain Multiple Sequence Subgroups with Distinctive Chloroplast-Targeting Sequence Motifs. Plant Cell 20: 1603-1622.

Lee, D.W., Yoo, Y.-J., Razzak, Md.A., and Hwang, I. (2018). Prolines in Transit Peptides Are Crucial for Efficient Preprotein Translocation into Chloroplasts. Plant Physiol. 176: 663677.

Lee, J., Lee, H., Kim, J., Lee, S., Kim, D.H., Kim, S., and Hwang, I. (2011). Both the Hydrophobicity and a Positively Charged Region Flanking the C-Terminal Region of the Transmembrane Domain of Signal-Anchored Proteins Play Critical Roles in Determining Their Targeting Specificity to the Endoplasmic Reticulum or Endosymbiotic Organelles in Arabidopsis Cells. Plant Cell 23: 1588-1607. 
Lee, J.-Y. (2015). Plasmodesmata: a signaling hub at the cellular boundary. Curr Opin Plant Biol 27: $133-140$.

Li, B.-C., Zhang, C., Chai, Q.-X., Han, Y.-Y., Wang, X.-Y., Liu, M.-X., Feng, H., and Xu, Z.-Q.

Li, T., Xiao, Z., Li, H., Liu, C., Shen, W., and Gao, C. (2020). A Combinatorial Reporter Set to Visualize the Membrane Contact Sites Between Endoplasmic Reticulum and Other Organelles in Plant Cell. Front. Plant Sci. 11: 1280.

Lim, G.-H., Shine, M.B., de Lorenzo, L., Yu, K., Cui, W., Navarre, D., Hunt, A.G., Lee, J.-Y., Kachroo, A., and Kachroo, P. (2016). Plasmodesmata Localizing Proteins Regulate Transport and Signaling during Systemic Acquired Immunity in Plants. Cell Host Microbe 19: 541-549.

Mine, A., Seyfferth, C., Kracher, B., Berens, M.L., Becker, D., and Tsuda, K. (2018). The

Lu, H., Liu, Y., and Greenberg, J.T. (2005). Structure-function analysis of the plasma

Mészáros, T., Helfer, A., Hatzimasoura, E., Magyar, Z., Serazetdinova, L., Rios, G., Bardóczy, V., Teige, M., Koncz, C., Peck, S., and Bögre, L. (2006). The Arabidopsis MAP kinase kinase MKK1 participates in defence responses to the bacterial elicitor flagellin. The Plant Journal 48: 485-498. Defense Phytohormone Signaling Network Enables Rapid, High-Amplitude Transcriptional Reprogramming during Effector-Triggered Immunity. Plant Cell 30: 1199-1219.

Mohr, P.G. and Cahill, D.M. (2007). Suppression by ABA of salicylic acid and lignin

Nielsen, H., Engelbrecht, J., Brunak, S., and von Heijne, G. (1997). Identification of prokaryotic accumulation and the expression of multiple genes, in Arabidopsis infected with Pseudomonas syringae pv. tomato. Funct Integr Genomics 7: 181-191.

Mittler, R., Vanderauwera, S., Gollery, M., and Van Breusegem, F. (2004). Reactive oxygen gene network of plants. Trends in Plant Science 9: 490-498. and eukaryotic signal peptides and prediction of their cleavage sites. Protein Eng 10: 1-6.

Peltier, J.-B., Ytterberg, A.J., Sun, Q., and van Wijk, K.J. (2004). New functions of the thylakoid membrane proteome of Arabidopsis thaliana revealed by a simple, fast, and versatile fractionation strategy. J Biol Chem 279: 49367-49383.

Pieterse, C.M., van Wees, S.C., Hoffland, E., van Pelt, J.A., and van Loon, L.C. (1996). Systemic resistance in Arabidopsis induced by biocontrol bacteria is independent of salicylic acid accumulation and pathogenesis-related gene expression. Plant Cell 8: 12251237. 
Pieterse, C.M., van Wees, S.C., van Pelt, J.A., Knoester, M., Laan, R., Gerrits, H., Weisbeek, P.J., and van Loon, L.C. (1998). A novel signaling pathway controlling induced systemic resistance in Arabidopsis. Plant Cell 10: 1571-1580.

Pieterse, C.M.J., Zamioudis, C., Berendsen, R.L., Weller, D.M., Van Wees, S.C.M., and Bakker, P.A.H.M. (2014). Induced systemic resistance by beneficial microbes. Annu Rev Phytopathol 52: 347-375.

Pitzschke, A., Datta, S., and Persak, H. (2014). Salt stress in Arabidopsis: lipid transfer protein AZI1 and its control by mitogen-activated protein kinase MPK3. Mol Plant 7: 722-738.

Qutob, D. et al. (2006). Phytotoxicity and Innate Immune Responses Induced by Nep1-Like Proteins. Plant Cell 18: 3721-3744.

Rekhter, D., Lüdke, D., Ding, Y., Feussner, K., Zienkiewicz, K., Lipka, V., Wiermer, M., Zhang, Y., and Feussner, I. (2019). Isochorismate-derived biosynthesis of the plant stress hormone salicylic acid. Science 365: 498-502.

Seguel, A., Jelenska, J., Herrera-Vásquez, A., Marr, S.K., Joyce, M.B., Gagesch, K.R., Shakoor, N., Jiang, S.-C., Fonseca, A., Wildermuth, M.C., Greenberg, J.T., and Holuigue, L. (2018). PROHIBITIN3 Forms Complexes with ISOCHORISMATE SYNTHASE1 to Regulate Stress-Induced Salicylic Acid Biosynthesis in Arabidopsis. Plant Physiol 176: $2515-2531$.

Serrano, M., Wang, B., Aryal, B., Garcion, C., Abou-Mansour, E., Heck, S., Geisler, M., Mauch, F., Nawrath, C., and Métraux, J.-P. (2013). Export of Salicylic Acid from the Chloroplast Requires the Multidrug and Toxin Extrusion-Like Transporter EDS5. Plant Physiol. 162: $1815-1821$.

Siemens, J., Keller, I., Sarx, J., Kunz, S., Schuller, A., Nagel, W., Schmülling, T., Parniske, M., and Ludwig-Müller, J. (2006). Transcriptome Analysis of Arabidopsis Clubroots Indicate a Key Role for Cytokinins in Disease Development. MPMI 19: 480-494.

Snider, C., Jayasinghe, S., Hristova, K., and White, S.H. (2009). MPEx: A tool for exploring membrane proteins. Protein Science 18: 2624-2628.

Stringlis, I.A., Proietti, S., Hickman, R., Van Verk, M.C., Zamioudis, C., and Pieterse, C.M.J. (2018). Root transcriptional dynamics induced by beneficial rhizobacteria and microbial immune elicitors reveal signatures of adaptation to mutualists. Plant J 93: 166-180.

Thilmony, R., Underwood, W., and He, S.Y. (2006). Genome-wide transcriptional analysis of the Arabidopsis thaliana interaction with the plant pathogen Pseudomonas syringae pv. tomato DC3000 and the human pathogen Escherichia coli O157:H7. The Plant Journal 46: $34-53$.

Tomizioli, M. et al. (2014). Deciphering Thylakoid Sub-compartments using a Mass Spectrometry-based Approach. Mol Cell Proteomics 13: 2147-2167. 
Toulmay, A. and Prinz, W.A. (2011). Lipid transfer and signaling at organelle contact sites: the tip of the iceberg. Curr Opin Cell Biol 23: 458-463.

Tripp, J., Inoue, K., Keegstra, K., and Froehlich, J.E. (2007). A novel serine/proline-rich domain in combination with a transmembrane domain is required for the insertion of AtTic 40 into the inner envelope membrane of chloroplasts. Plant J. 52: 824-838.

Wintermans, P.C.A., Bakker, P.A.H.M., and Pieterse, C.M.J. (2016). Natural genetic variation in Arabidopsis for responsiveness to plant growth-promoting rhizobacteria. Plant Mol Biol 90: 623-634.

Yu, K., Soares, J.M., Mandal, M.K., Wang, C., Chanda, B., Gifford, A.N., Fowler, J.S., Navarre, D., Kachroo, A., and Kachroo, P. (2013). A Feedback Regulatory Loop between G3P and Lipid Transfer Proteins DIR1 and AZI1 Mediates Azelaic-Acid-Induced Systemic Immunity. Cell Reports 3: 1266-1278.

Yu, Z. et al. (2020). The Brassicaceae-specific secreted peptides, STMPs, function in plant growth and pathogen defense. J Integr Plant Biol 62: 403-420.

Zamioudis, C., Hanson, J., and Pieterse, C.M.J. (2014). $\beta$-Glucosidase BGLU42 is a MYB72dependent key regulator of rhizobacteria-induced systemic resistance and modulates iron deficiency responses in Arabidopsis roots. New Phytol 204: 368-379.

Zamioudis, C., Mastranesti, P., Dhonukshe, P., Blilou, I., and Pieterse, C.M.J. (2013). Unraveling root developmental programs initiated by beneficial Pseudomonas spp. bacteria. Plant Physiol 162: 304-318.

Zipfel, C. (2008). Pattern-recognition receptors in plant innate immunity. Curr. Opin. Immunol. 20: $10-16$.

Zoeller, M., Stingl, N., Krischke, M., Fekete, A., Waller, F., Berger, S., and Mueller, M.J. (2012). Lipid Profiling of the Arabidopsis Hypersensitive Response Reveals Specific Lipid Peroxidation and Fragmentation Processes: Biogenesis of Pimelic and Azelaic Acid. Plant Physiol. 160: 365-378.

Zuegge, J., Ralph, S., Schmuker, M., McFadden, G.I., and Schneider, G. (2001). Deciphering apicoplast targeting signals - feature extraction from nuclear-encoded precursors of Plasmodium falciparum apicoplast proteins. Gene 280: 19-26. 


\section{$853 \quad$ Figure legends}

854 Figure 1. Targeting of Arabidopsis bipartite signal proteins to plastids. (A) Schematic of N-

855 terminal bipartite signal of Arabidopsis HyPRPs/Apicomplexan proteins predicted by PATS. SP-

856 like is signal-peptide like and cTP-like is chloroplast transit-peptide like sequence. (B) The

857 percentage prediction output of PATS algorithm for each chromosome of Arabidopsis. The full-

858 length sequences of Arabidopsis proteome (ATpepTAIR10) were analyzed by PATS for the

859 predicted localization (\%) to plastids. (C) Left panels: Laser scanning confocal micrographs of

860 epidermal cells showing localization of non-HyPRP:GFP proteins encoded by the indicated

861 genes transiently expressed in $N$. benthamiana leaves under Dex control. Samples were imaged

862 21-24h after infiltration with $30 \mu \mathrm{M}$ Dex. White arrowheads indicate chloroplasts (shown in

863 insets). Scale bar is $20 \mu \mathrm{m}$. Right Panel: Immunoblots of total (T) and chloroplast (Chl) fractions

864 of N. benthamiana leaves transiently expressing At5g54170-encoded and STMP6 GFP-tagged

865 proteins. Chloroplast fractions were isolated from leaves $21 \mathrm{~h}$ after treatment with $30 \mu \mathrm{M}$ Dex.

866 Signal was observed using anti-GFP antibody. ns is non-specific band; arrows indicate specific

867 band. Coomassie blue (CB) staining shows the protein loading. (D) Targeting prediction of

868 Arabidopsis HyPRPs by iterative TargetP/ChloroP and PATS algorithms. For iTargetP/iChloroP,

869 the sequences without N-terminal SP-like region were used. The proteins with targeting studied

870 herein are in bold. The asterisk indicates AZI1, a variant of signal-anchored protein, where SP-

871 like domain is a transmembrane (TMD) (Cecchini et al., 2020). HyPRPs not predicted by either

872 method to be plastid-localized are in the lower section of the panel.

873 Figure 2. Subcellular localization of HyPRP:GFPs in N. benthamiana. (A) Laser scanning

874 confocal micrographs of epidermal cells showing localization of various HyPRP:GFP proteins

875 transiently expressed in N. benthamiana leaves under Dex-inducible promoter. Samples were 


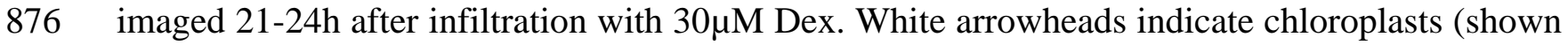

877 in insets), red arrowheads show plasmodesmata-like structures/plasma membrane and yellow

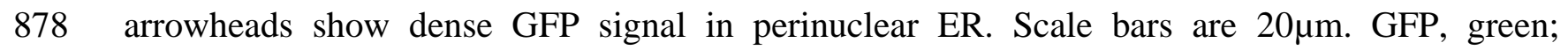

879 chloroplast autofluorescence, blue. (B) Immunoblots of total (T) and chloroplast (chl) fractions

880 of $N$. benthamiana leaves transiently expressing a subset of HyPRP:GFP proteins shown in (A).

881 Chloroplast fractions were isolated from leaves $21 \mathrm{~h}$ after treatment with $30 \mu \mathrm{M}$ Dex. Signal was

882 observed using anti-GFP or anti-HA antibody. Coomassie blue (CB) or Ponceau S (PS) staining

883 shows the protein loading. (C) Normalized total spectra of peptides matching AZL3 in

884 chloroplast membranes of constitutively defense active ACD6-1HA and WT. PHB3, a known

885 chloroplast envelope protein is shown as control (Seguel et al., 2018). Total spectra

886 normalization was analyzed by Scaffold software for each sample (WT or ACD6-1HA).

887 Figure 3. Chloroplast localization of HyPRP:GFP proteins in Arabidopsis transgenic lines.

888 Laser scanning confocal micrographs of epidermal cells showing chloroplast localization of

889 indicated GFP tagged HyPRPs in 9-12 day-old Arabidopsis transgenic seedlings (hypocotyl).

890 Plants were sprayed with $30 \mu \mathrm{M}$ Dex plus $0.04 \%$ Tween 20, 21-24h prior to imaging.

891 Fluorescence intensity profiles in the merged images show the overlap of GFP (green) and

892 chlorophyll (Chyll, blue) signal intensities along solid white lines (indicated by the red

893 arrowheads). Note the overlap of GFP (green) and chlorophyll (Chyll, blue) intensities along

894 chloroplast peripheries. The plastid-targeted GFP line (cTP-GFP) (Jiang et al., 2021) and

895 untransformed WT (Col-0) serve as positive and negative controls, respectively, for this

896 experiment. The images were analyzed by ImageJ (Fiji). Similar results were observed in two

897 independent experiments. White arrowheads indicate chloroplast localization. Scale bar is $5 \mu \mathrm{m}$.

898 GFP, green; chloroplast autofluorescence, blue. 
900 Immunoblots of total (T), membrane (M) and soluble (S) fractions of isolated chloroplasts from

901 N. benthamiana leaves transiently expressing AZL14, AZL13, AZL2 and ELP tagged with GFP

902 at C-terminal under Dex-inducible promoter. HyPRPs were detected with $\alpha$-GFP antibody. $\alpha$ -

903 Tic110 and $\alpha$-cpHSP70 antibodies were used to detect the markers of inner envelope membrane,

904 Tic110 and stroma, HSP70. (B) Immunoblots of intact chloroplast fractions expressing

905 HyPRP:GFPs as in (A) treated with buffer (-) or thermolysin (+). The digestion of HyPRP:GFPs

906 by thermolysin was evaluated by $\alpha$-GFP antibody. Due to the higher observed expression of

907 ELP:GFP compared to other HyPRP:GFPs, 1/4X total protein was loaded for this sample. A low

908 exposure for ELP:GFP with $\alpha$-GFP antibody is shown. The antibodies for OEM protein Toc75

909 and stromal HSP70 were used to detect the non-digestible controls. These experiments were

910 repeated two-three times with similar results.

911 Figure 5. Subcellular localization of the truncated versions of HyPRP:GFPs in $N$.

912 benthamiana. (A) Schematic of a HyPRP showing N-terminal SP-like or HD, PRR and the C-

913 terminal $8 \mathrm{CM} / \mathrm{LTP}$ domain. The $\triangle \mathrm{PRR}$ and $\triangle \mathrm{LTP}$ versions are also depicted. (B) Confocal

914 micrographs showing the localization of $\triangle \mathrm{PRR}$ mutants of GFP-tagged HyPRPs in epidermal

915 cells under Dex-inducible promoter. Lower panels show the enlarged views of chloroplasts. (C)

916 Immunoblots of total (T) and chloroplast (Chl) fractions from N. benthamiana leaves transiently

917 expressing HyPRP:GFP proteins as in (B). GFP-tagged HyPRPs were detected by $\alpha$-GFP

918 antibody. ns is non-specific band; arrow indicates specific band. The same blots stained with

919 Coomassie blue (CB) show protein loading. Similar results were observed in two or more

920 independent experiments for AZL14, AZL13 and ELP. For AZL2, the result of one experiment

921 is shown. (D) Confocal micrograph of epidermal cells and immunoblot of total (T) and 
922 chloroplast (Chl) fraction showing the subcellular localization of GFP-tagged ELP: $\Delta$ LTP. Inset

923 shows signal associated with chloroplast. For the micrographs, GFP is green; chloroplast

924 autofluorescence is blue. All scale bars are $20 \mu \mathrm{m}$.

925 Figure 6. Expression, predicted co-expression and plastid envelope localization of $H y P R P s$.

926 (A) Network co-expression map of the HyPRP genes using ATTED-II (version 11.0;

927 https://atted.jp/). Solid lines indicate co-expression between the genes included within the top 20

928 with higher score (Logit Score). Line thickness indicates the co-expression score. Condition-

929 independent and condition-specific co-expression data is depicted. Green circles indicate

930 HyPRPs targeted to the plastid envelope found in this and our previous (Cecchini et al., 2015)

931 study. Dotted green circle indicates DRN1 plastid targeting evidenced in this and other studies

932 (Peltier et al., 2004; Tomizioli et al., 2014). Light green circles indicate partial targeting to

933 plastids. Inside the dashed box are the genes with no co-expression or a Logit score lower than 3.

934 (B) Transcript levels of HyPRPs in PmaDG6-infected WT (Col-0) leaves 18hpi. PR1 induction

935 shows that the defense response was activated in these plants. (C) Transcript levels of AZL3 in

936 constitutively defense active mutant ACD6-1HA. In (B and C) EF1 $\alpha$ was used as an internal

937 reference. Error bars indicate SEM from two biological replicates and two technical replicates.

$938 * \mathrm{p}<0.05$, student's t-test.

939 Figure 7. SAR in Arabidopsis HyPRP mutants. (A, C) Growth of virulent bacteria PmaDG3

940 in systemic leaves of WT (Col-0) and indicated mutants three days after infection. The plants

941 were previously immunized in local leaves with avirulent PmaDG6 or mock treated, 2 days prior

942 to the secondary infection. The mean c.f.u. of two-three independent experiments are plotted. (B)

943 Response gain of SAR due to PmaDG6 immunization in (A). (D) Response gain of SAR due to

944 PmaDG6 immunization in (C). (E) PR1 induction at the indicated time points in the uninfected 
945 systemic leaves of plants that were locally mock treated or immunized with PmaDG6. (F)

946 Densitometric quantification of PR1 levels in immunized samples shown in (E). The PR1 levels

947 are relative to the non-specific band in Coomassie blue (CB) stained membrane. The mean from

948 two replicates is shown. Quantification was done by ImageJ (Fiji). (G) Growth of virulent

949 bacteria PmaDG3 in systemic leaves of WT (Col-0) and Dex-inducible complementation lines,

950 three days after infection. The plants were mock treated or immunized as in (A and $\mathbf{C}) .30 \mu \mathrm{M}$

951 Dex plus $0.04 \%$ Tween 20 was sprayed on local and systemic leaves $21 \mathrm{~h}$ before local and distal

952 infection, respectively. (H) RT-PCR of the indicated HyPRP transcripts in Dex-inducible

953 complementation lines. Leaves were collected $21 \mathrm{~h}$ after treatment with $30 \mu \mathrm{M}$ Dex (+) or no

954 treatment (-). Expression of EFl $\alpha$ was used as control. (I) Response gain of SAR due to

955 PmaDG6 immunization in $(\mathbf{G})$. In the graphs showing 'response gain', error bars indicate

956 uncertainties in the experiment; in all other graphs error bars show SEM. Different letters above

957 the bars indicate statistically significant difference. Anova, SNK test or Student's test, $\mathrm{p}<0.05$

958 Figure 8. ISR in HyPRPs mutants in Arabidopsis. (A) ISR was assayed by quantification of

959 virulent bacteria PmaDG3 in leaves of plants, three days after infection. The roots of the plants

960 were mock-treated or inoculated with $P$. simiae WCS417, 15-18 days before challenging the

961 leaves with PmaDG3. Error bars show SEM. (B) Response gain of ISR due to P. simiae WCS417

962 immunization in roots of plants in (A). Error bars indicate uncertainties in the experiment. The

963 mean c.f.u. of two independent experiments are plotted. Different letters above the bars indicate

964 statistically significant difference by anova, SNK test, $\mathrm{p}<0.05$

965 Figure 9. Growth promotion and root-colonization of HyPRP mutants by $P$. simiae

966 WCS417. (A) Representative images of WT (Col-0) and indicated mutants grown on agar plates

967 without sugar and inoculated with mock or P. simiae WCS417 (WCS417). 15 day-old seedlings, 
10dpi with WCS417 or mock are shown. (B) Fresh weight, (C) number of lateral roots and (D)

969 primary root length in plants inoculated with mock or WCS417 as in $(\mathbf{A}), \mathrm{n}=15-20$ plants.

970 Combined data from three independent experiments is plotted. (E) Representative images of WT

971 (Col-0) plants grown hydroponically in 48-well plates before (12 day-old) and 7dpi (19 day-old)

972 with WCS417. (F) Number of c.f.u. of WCS417 in the wells or attached to the roots of WT and

973 mutants. 'No plant' represents the number of c.f.u. of WCS417 in the well-media without any

974 plant present. $\mathrm{n}=20-24$ plants. In all graphs error bars are SEM and different letters above bars

975 indicate statistical differences by anova, SNK test. $\mathrm{p}<0.05$.

976 Figure 10. Speculative model and summary of roles of HyPRPs in microbial interactions.

977 (A) Cellular localization and proposed roles of HyPRPs in signal mobilization. Green color

978 indicates organelles and structures in cells where HyPRPs are found. Arrows show the potential

979 mobilization of signals by HyPRPs, either within a cell or locally near the infection (dashed line

980 and $*$ ) or the long-distance mobilization of signals to distal tissue. Although leaf epidermal cells

981 are depicted, similar localization patterns are expected for root cells. HyPRPs with * have roles

982 in local responses to microbes (root or leaf colonization); most also have roles in longer distance

983 responses as well (see part B). Data from this and previous work (Cecchini et al., 2015, 2020;

984 Peltier et al., 2004; Tomizioli et al., 2014) is summarized. The cell on the left shows HyPRPs

985 that likely locate to plastid thylakoids and/or OEMs as well as ER and PM/PD. HyPRPs that fit

986 the left pattern, AZL3* and DRN1*, have positive roles. The cell on the right shows HyPRPs

987 that locate to plastid OEMs as well as ER and/or PM/PDs. HyPRPs that fit the right pattern have

988 positive roles (AZI1, EARLI1, AZL2, AZL13, and AZL14), a mainly negative role (ELP) or

989 have unknown roles (CWLP, DHyPRP, AZI3, AZI4) in microbial responses. (B) Schematic of

990 the steps at the whole plant level that are affected by one or more HyPRPs: 1, leaf colonization 
991 by pathogen (DRN1, positive role); 2, increased immunity to distal infections after an

992 immunizing infection at site 1 (SAR: AZI1, EARLI1,AZL2, AZL3, AZL13, positive roles; ELP,

993 negative role); 3, growth of beneficial bacteria in association with roots (AZL3, AZL13, AZL14,

994 positive roles); 4, stimulation of primary root growth in response to beneficial bacteria

995 colonization of the root (ELP, AZL14, positive roles); 5, stimulation of lateral roots in response

996 to beneficial bacteria colonization of the root (AZL13, AZL14, positive roles; ELP, negative

997 role); 6, stimulation of whole plant growth by root colonization by beneficial bacteria (AZL3,

998 AZL13, AZL14, positive roles; ELP, negative role); 7, increased immunity of aerial tissue (ISR

999 after root colonization by beneficial bacteria: AZI1, EARLI1, AZL3, AZL13, AZL14, positive

1000 roles).

1001

1002 Supplementary Figure Legends

1003 Supplementary Figure S1. Over-represented GO terms for biological processes in PATS

1004 plastid predicted proteins, location of $\mathbf{H y P R P s}$ in Arabidopsis genome and GFP cleavage in

1005 DRN1. (A) The most representative and significant GO terms sorted by fold enrichment are

1006 plotted. The size of dot indicates the number of genes associated with the process and the color

1007 of dot indicates the significance of the enrichment $\left\{-\log _{10}(\right.$ FDR-corrected $P$-values $\left.)\right\}$. The

1008 vertical dashed line represents a fold enrichment of 1. (B) Location of HyPRP genes depicted in

1009 Arabidopsis genome, made with the TAIR chromosome map tool

1010 (https://www.arabidopsis.org/jsp/ChromosomeMap/tool.jsp). (C) Confocal micrograph of

1011 epidermal cells and immunoblotting of total (T) fraction of $N$. benthamiana leaves transiently

1012 expressing DRN1-GFP showing cleavage of GFP. White arrowhead shows cytoplasm and white 
1013 arrow indicates nucleus. Scale bar is $20 \mu \mathrm{m}$. Asterisk shows the free GFP detected by anti-GFP

1014 antibody. rbcL is the non-specific rubisco large subunit.

1015

1016

1017 Supplementary Figure S2. ClustalW alignment of Arabidopsis HyPRPs. The bars above the

1018 alignment indicate the putative SP-like or HD, PRR and 8CM/LTP domain. Black and grey

1019 boxes indicate identical or similar sequences, respectively. Red asterisks show the eight

1020 conserved Cys residues. AZL4 and AZL8 were excluded due to relatively larger sizes. ClustalW

1021 and BoxShade (Expasy tools) were used for the alignment.

1022

1023 Supplementary Figure S3. Local disease resistance of HyPRP mutants against

1024 Pseudomonas syringae pv. maculicola strains. Wild-type (Col-0) and indicated HyPRP mutants

1025 were infected with (A) PmaDG3 (virulent strain) and (B) PmaDG6 containing avrRpt2 (SAR-

1026 inducing strain) at $\mathrm{OD}_{600}=0.0001$ and growth measured 3 days post infiltration. Error bars

1027 indicate SEM. Figures represent the combined data from two independent experiments with n=7-

10288 for each replicate. Anova (SNK test) showed no statistically significant difference between WT

1029 and $H y P R P$ mutants.

1030

1031 Supplementary Figure S4. SAR in elp-2 allelic mutant and Dex-inducible complementation

1032 lines. SAR assay showing the growth of virulent bacteria PmaDG3 in systemic leaves of (A) elp-

10332 mutants and (B) Dex-inducible complementation lines (\#2), three days after infection. The

1034 plants were immunized in local leaves with avirulent PmaDG6 or mock treated 2 days prior to

1035 secondary challenge. The mean c.f.u. of one experiment is shown. (C) RT-PCR of the indicated 
1036 Dex line (\#2). Leaves were collected $21 \mathrm{~h}$ after treatment with $30 \mu \mathrm{M}$ Dex plus $0.04 \%$ Tween 20

1037 (+) or no treatment (-). Expression of EFI $\alpha$ was used as control. In (A, B), error bars show SEM.

1038 Different letters above the bars indicate statistically significant difference by anova, SNK test, $1039 \mathrm{p}<0.05$.

1040 
Table 1. Expression changes of Arabidopsis HyPRPs to WCS417, pathogens and PAMPs

\begin{tabular}{|c|c|c|}
\hline HyPRP & \begin{tabular}{|c|} 
Pathogen/PAMP/Beneficial microbe- \\
related
\end{tabular} & References \\
\hline At1g12090 (ELP) & $\begin{array}{c}\text { CalCuV } \downarrow, \text { Pst } \downarrow \text {, PAMPs } \downarrow, \text { Psm } \downarrow, \\
\text { flg22 (roots) }\end{array}$ & $\begin{array}{l}\text { (Ascencio-Ibáñez et al., 2008; } \\
\text { Baum et al., 2019; Stringlis et al., } \\
\text { 2018; Thilmony et al., 2006) }\end{array}$ \\
\hline At1g12100 (AZL1) & flg $22 \downarrow$ (roots) & (Stringlis et al., 2018) \\
\hline At1g62500 (DEG27) & WCS417个(roots), flg22 & (Stringlis et al., 2018) \\
\hline At1g62510 (AZL2) & 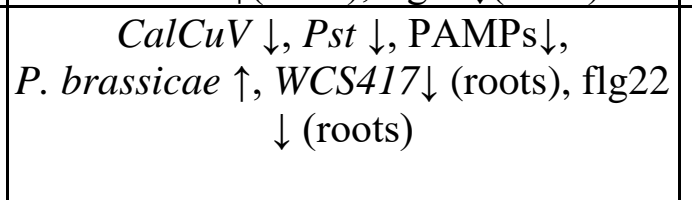 & $\begin{array}{l}\text { (Ascencio-Ibáñez et al., 2008; } \\
\text { Siemens et al., 2006; Stringlis et } \\
\text { al., 2018; Thilmony et al., 2006; } \\
\text { Zamioudis et al., 2014) }\end{array}$ \\
\hline At2g109 & $\begin{array}{l}\text { CalCuV } \downarrow, \text { Pst } \downarrow, \text { PAM } \\
W C S 417 \downarrow \text { (roots), flg }\end{array}$ & $\begin{array}{l}\text { (Ascencio-Ibáñez et al., 2008; } \\
\text { Baum et al., 2019; Stringlis et al., } \\
\text { 2018; Thilmony et al., 2006) }\end{array}$ \\
\hline At2g451 & $\begin{array}{r}\text { CalCuV } \downarrow, \text { Pst } \downarrow, \mathrm{P} \\
\text { cinerea } \downarrow \text { 年 } \mathrm{fl}\end{array}$ & $\begin{array}{l}\text { (Ascencio-Ibáñez et al., 2008; } \\
\text { Dhar et al., 2020; Stringlis et al., } \\
\text { 2018; Thilmony et al., 2006) }\end{array}$ \\
\hline$\overline{\text { At3g2212 }}$ & $\begin{array}{r}\text { CalCuV } \downarrow \text {, PAMP } \\
D C 3000 / \text { avrR }\end{array}$ & $\begin{array}{l}\text { (Ascencio-Ibáñez et al., 2008; } \\
\text { Thilmony et al., 2006; Mine et al., } \\
\text { 2018) }\end{array}$ \\
\hline At3g2 & $\sqrt{2}(10$ & 1., 2018) \\
\hline At4g00 & $17 \downarrow$ (ros & \\
\hline At4g1247 & $\begin{array}{r}P s m \uparrow, \text { PAMPs } \uparrow, W \\
\text { flg22PA } \uparrow\end{array}$ & $\begin{array}{l}\text { (Cecchini et al., 2020; Jung et al., } \\
\text { 2009; Stringlis et al., 2018; } \\
\text { Zamioudis et al., 2014) }\end{array}$ \\
\hline $\begin{array}{c}\text { At4g12480 } \\
\text { (EARLI1) }\end{array}$ & $\begin{array}{r}\text { CalCuV } \uparrow, P s t \uparrow, \mathrm{P}_{2} \\
(1 \mathrm{~h} \mathrm{r}\end{array}$ & $\begin{array}{c}\text { (Ascencio-Ibáñez et al., 2008; } \\
\text { Gupta and Senthil-Kumar, 2017; } \\
\text { Stringlis et al., 2018; Thilmony et } \\
\text { al., 2006) }\end{array}$ \\
\hline At4g12 & $\begin{array}{r}\mathrm{CalCu} V \uparrow, \text { PAMPs } \\
\text { flg } 22 \uparrow\end{array}$ & $\begin{array}{l}\text { (Ascencio-Ibáñez et al., 2008; } \\
\text { Stringlis et al., 2018; Thilmony et } \\
\text { al., 2006; Zamioudis et al., 2014) }\end{array}$ \\
\hline At4g1250 & $\begin{array}{r}\text { PAMPs } \uparrow, P s t \uparrow, \\
\mathrm{N}\end{array}$ & $\begin{array}{l}\text { (Mohr and Cahill, 2007; Qutob et } \\
\text { al., 2006; Thilmony et al., 2006) }\end{array}$ \\
\hline At4g1251 & $\begin{array}{r}C a l C u V \downarrow, W C S 4 \\
\text { (ro }\end{array}$ & $\begin{array}{l}\text { (Ascencio-Ibáñez et al., 2008; } \\
\text { Stringlis et al., 2018) }\end{array}$ \\
\hline At4g12520 (AZI6) & 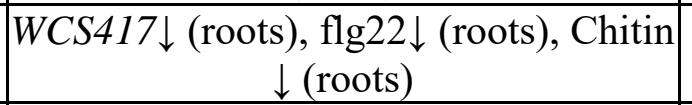 & (Stringlis et al., 2018) \\
\hline At4g12530 (AZI7) & $P$ Pst $\downarrow$ & 2013) \\
\hline At4g125 & $\mathrm{ts}), \mathrm{Cl}$ & \\
\hline & $\downarrow$ (roo & 8) \\
\hline & & \\
\hline At4g22460 (AZL7) & 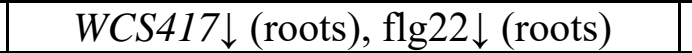 & (Stringlis et al., 2018) \\
\hline
\end{tabular}




\begin{tabular}{|c|c|c|}
\hline $\begin{array}{l}\text { At4g22470 } \\
(\mathrm{DHYPRP} 1)\end{array}$ & $\begin{array}{c}P s t \uparrow, \text { flg } 22 \uparrow, W C S 417 \uparrow \text { (roots), } \\
\text { flg } 22 \uparrow(\text { roots), flg22-PA } \uparrow \text { (roots), Chitin } \\
\uparrow(\text { roots) }\end{array}$ & $\begin{array}{l}\text { (Li et al., 2014; Mészáros et al., } \\
\text { 2006; Stringlis et al., 2018) }\end{array}$ \\
\hline At4g22485 (AZL8) & No known change & \\
\hline At4g22490 (AZL9) & $P s t \uparrow$ & (Gupta and Senthil-Kumar, 2017) \\
\hline At4g22517 (AZL10) & No known change & \\
\hline At4g22520 (AZL11) & No known change & \\
\hline At4g22610 (AZL12) & $\begin{array}{c}W C S 417 \downarrow \text { (roots), flg } 22 \downarrow \text { (roots), Chitin } \\
\uparrow(\text { roots) }\end{array}$ & (Stringlis et al., 2018) \\
\hline At5g46890 (AZL13) & $\begin{array}{c}W C S 417 \downarrow \text { (roots), flg22-417 } \downarrow \text { (roots), } \\
\text { flg22-PA } \downarrow \text { (roots) }\end{array}$ & (Stringlis et al., 2018) \\
\hline At5g46900 (AZL14) & $\begin{array}{c}W C S 417 \downarrow \text { (roots), flg22-417 } \downarrow \text { (roots), } \\
\text { flg22-PA } \downarrow \text { (roots) }\end{array}$ & (Stringlis et al., 2018) \\
\hline
\end{tabular}

CalCuV is Cabbage leaf curl virus, Psm is P. syrinage pv maculicola ES4326, Pst is $P$. syringae $1044 p v$ tomato DC3000, WCS417 is P. simiae WCS417, B. cinerea is Botrytis cinerea, PAMPs is 1045 pathogen-associated molecular patterns, $P$. brassiccae is Plasmodiophora brassicae, flg22-PA is 1046 flg22 from $P$. aeruginosa, NLP is (Nep1)-like protein 
A

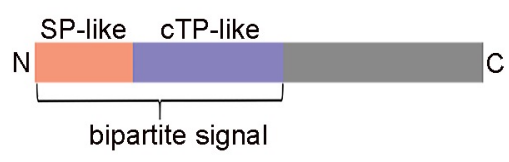

B

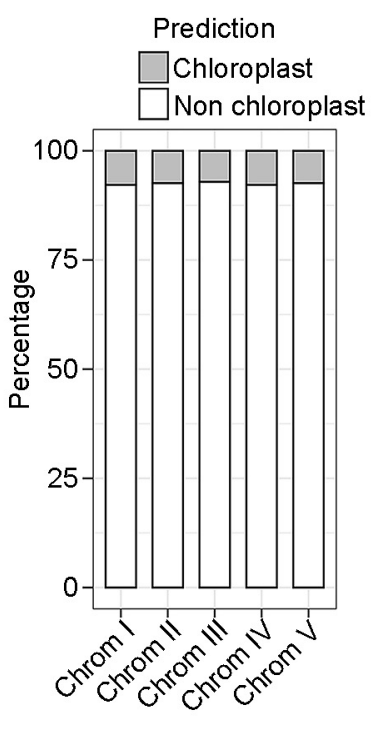

D

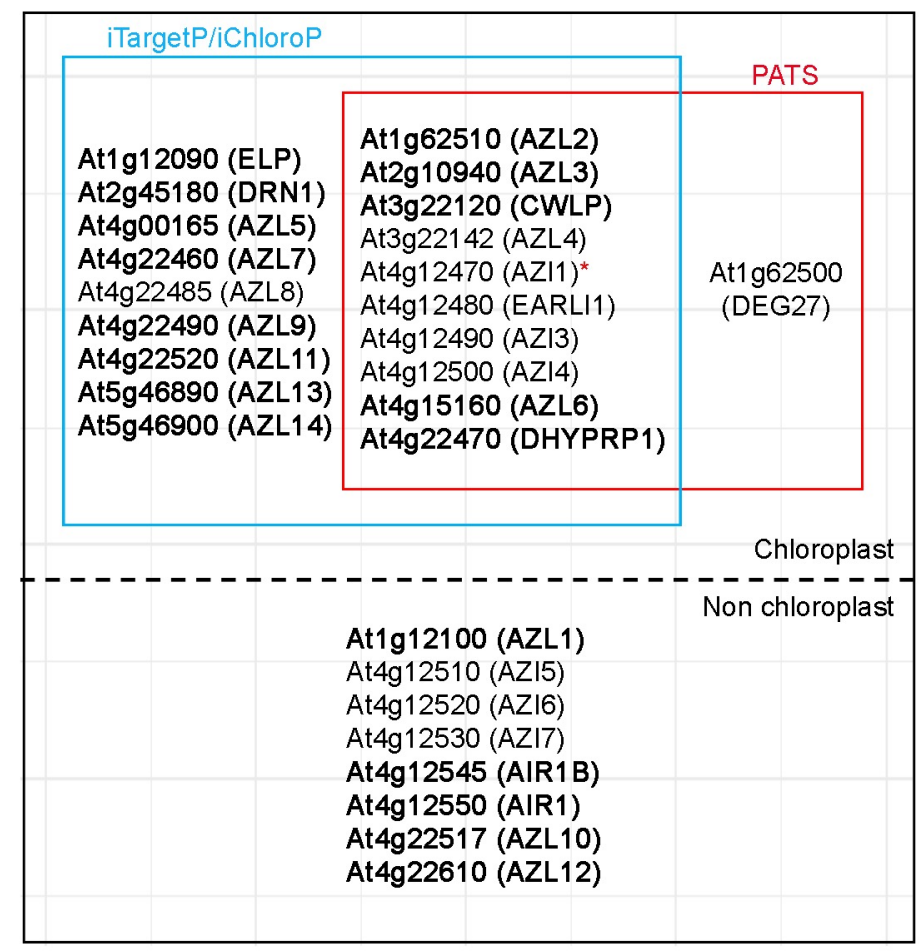

C

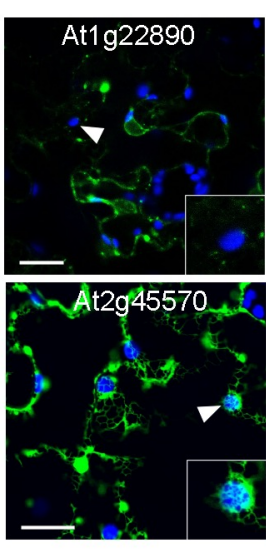

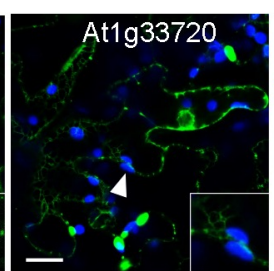

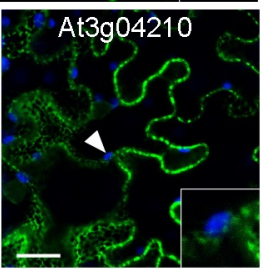

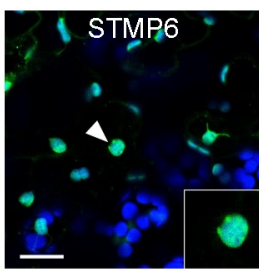

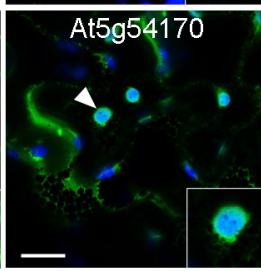

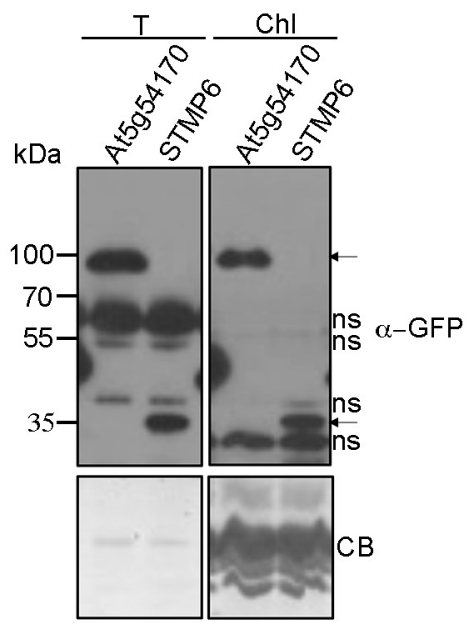


Figure 1. Targeting of Arabidopsis bipartite signal proteins to plastids. (A) Schematic of $\mathrm{N}$-terminal bipartite signal of Arabidopsis HyPRPs/Apicomplexan proteins predicted by PATS. SP-like is signal-peptide like and cTP-like is chloroplast transitpeptide like sequence. (B) The percentage prediction output of PATS algorithm for each chromosome of Arabidopsis. The full-length sequences of Arabidopsis proteome (ATpepTAIR10) were analyzed by PATS for the predicted localization (\%) to plastids. (C) Left panels: Laser scanning confocal micrographs of epidermal cells showing localization of non-HyPRP:GFP proteins encoded by the indicated genes transiently expressed in $N$. benthamiana leaves under Dex control. Samples were imaged 21-24h

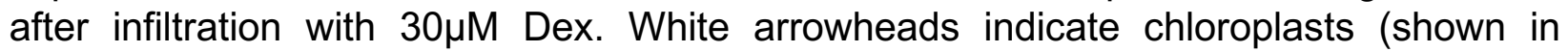
insets). Scale bar is $20 \mu \mathrm{m}$. Right Panel: Immunoblots of total (T) and chloroplast (Chl) fractions of $N$. benthamiana leaves transiently expressing At5g54170-encoded and STMP6 GFP-tagged proteins. Chloroplast fractions were isolated from leaves $21 \mathrm{~h}$ after treatment with $30 \mu \mathrm{M}$ Dex. Signal was observed using anti-GFP antibody. ns is nonspecific band; arrows indicate specific band. Coomassie blue (CB) staining shows the protein loading. (D) Targeting prediction of Arabidopsis HyPRPs by iterative TargetP/ChloroP and PATS algorithms. For iTargetP/iChloroP, the sequences without Nterminal SP-like region were used. The proteins with targeting studied herein are in bold. The asterisk indicates AZI1, a variant of signal-anchored protein, where SP-like domain is a transmembrane (TMD) (Cecchini et al., 2020). HyPRPs not predicted by either method to be plastid-localized are in the lower section of the panel. 


\section{A}
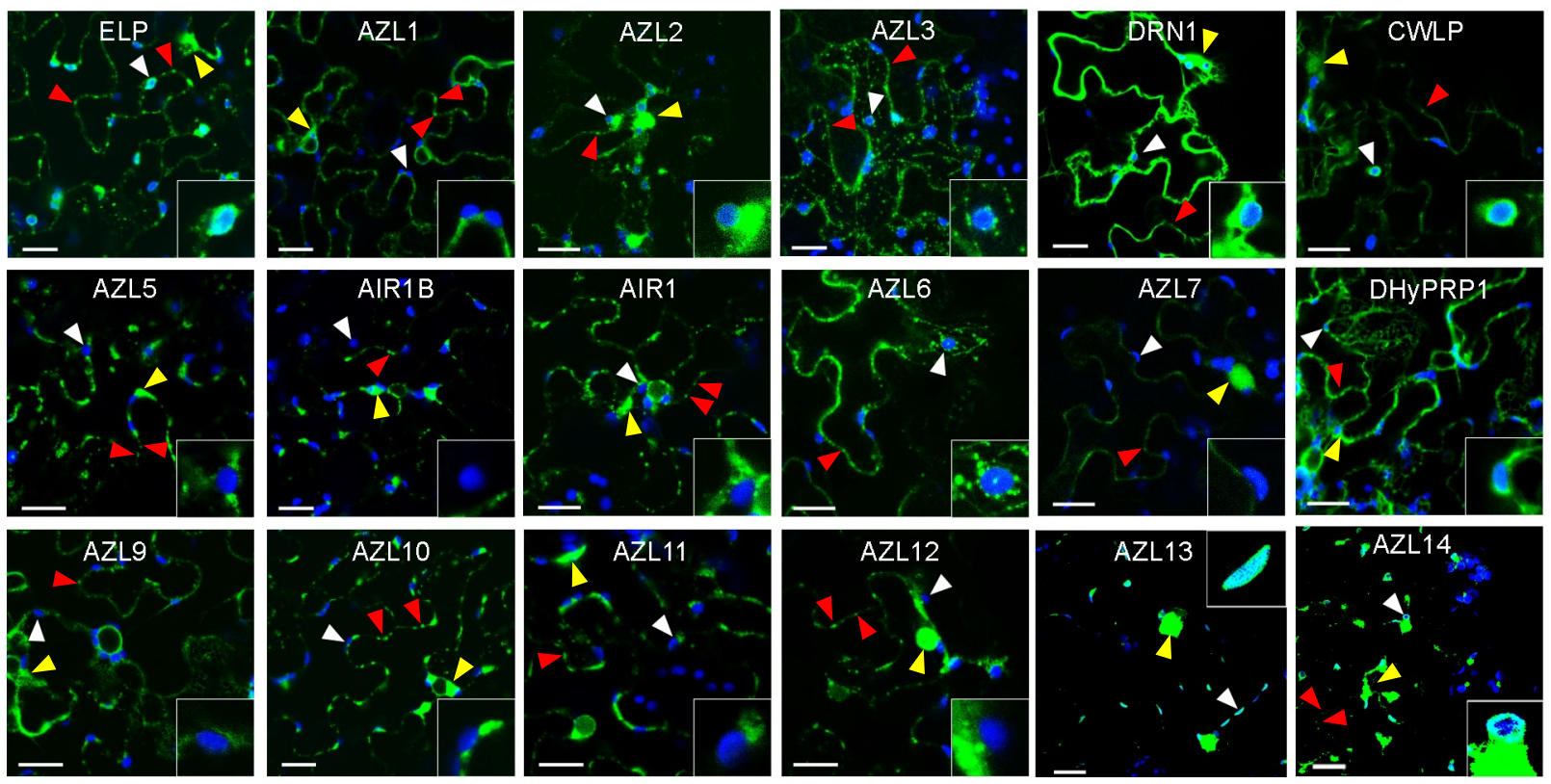

B
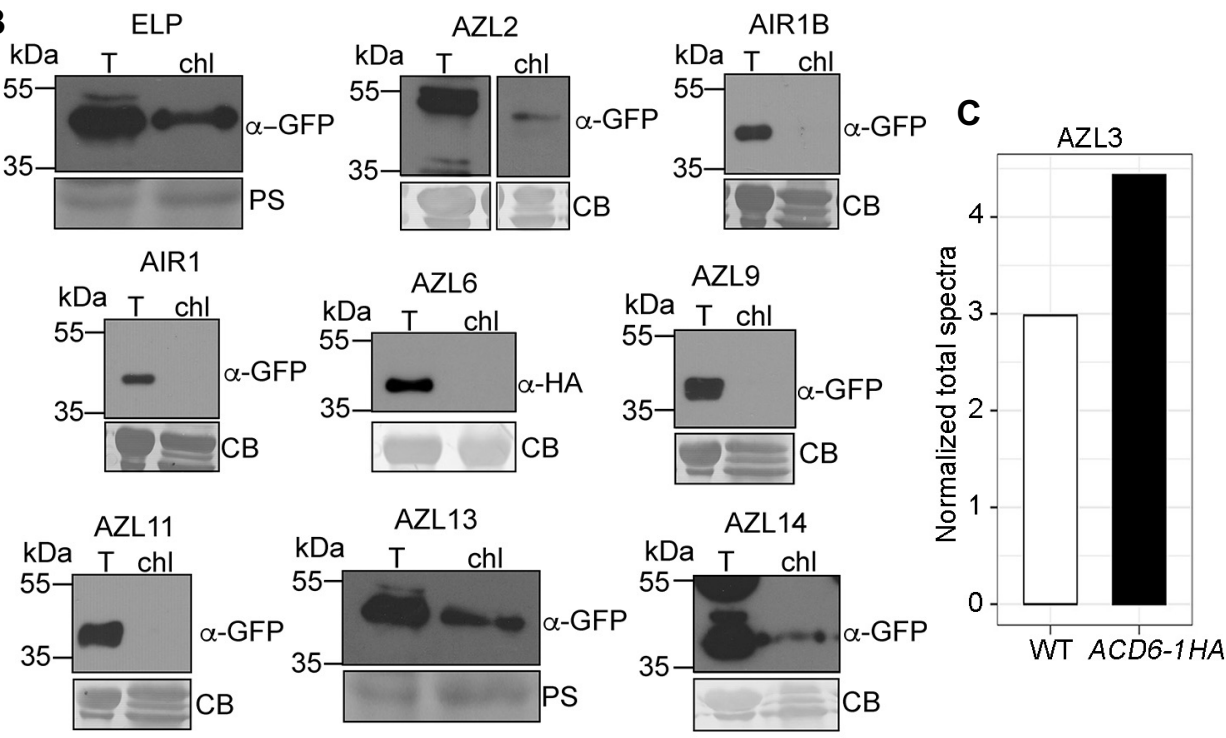

AZL13

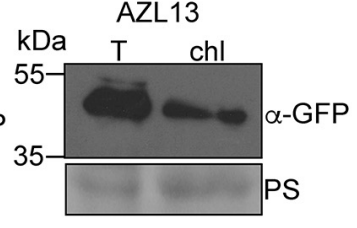

AZL14
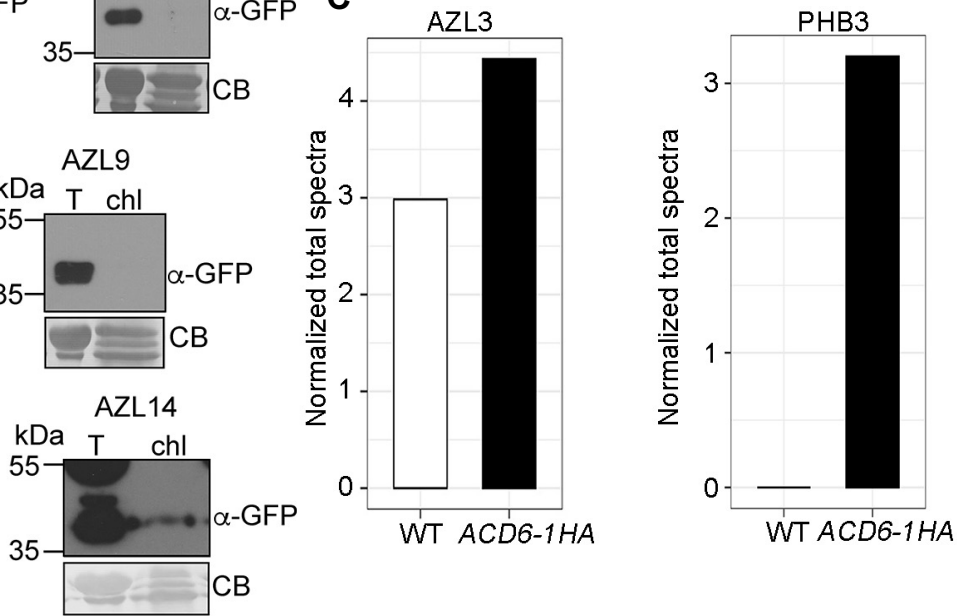

Figure 2. Subcellular localization of HyPRP:GFPs in $\mathbf{N}$. benthamiana. (A) Laser scanning confocal micrographs of epidermal cells showing localization of various HyPRP:GFP proteins transiently expressed in $N$. benthamiana leaves under Dex-

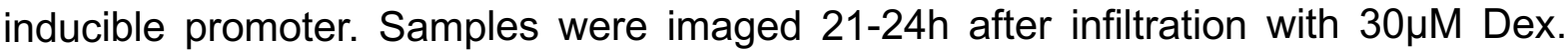
White arrowheads indicate chloroplasts (shown in insets), red arrowheads show plasmodesmata-like structures/plasma membrane and yellow arrowheads show dense GFP signal in perinuclear ER. Scale bars are 20 $\mu \mathrm{m}$. GFP, green; chloroplast autofluorescence, blue. (B) Immunoblots of total ( $T$ ) and chloroplast (chl) fractions of $N$. benthamiana leaves transiently expressing a subset of HyPRP:GFP proteins shown in (A). Chloroplast fractions were isolated from leaves $21 \mathrm{~h}$ after treatment with $30 \mu \mathrm{M}$ Dex. Signal was observed using anti-GFP or anti-HA antibody. Coomassie blue (CB) or Ponceau S (PS) staining shows the protein loading. (C) Normalized total spectra of peptides matching AZL3 in chloroplast membranes of constitutively defense active ACD6-1HA and WT. PHB3, a known chloroplast envelope protein is shown as control (Seguel et al., 2018). Total spectra normalization was analyzed by Scaffold software for each sample (WT or ACD6-1HA). 

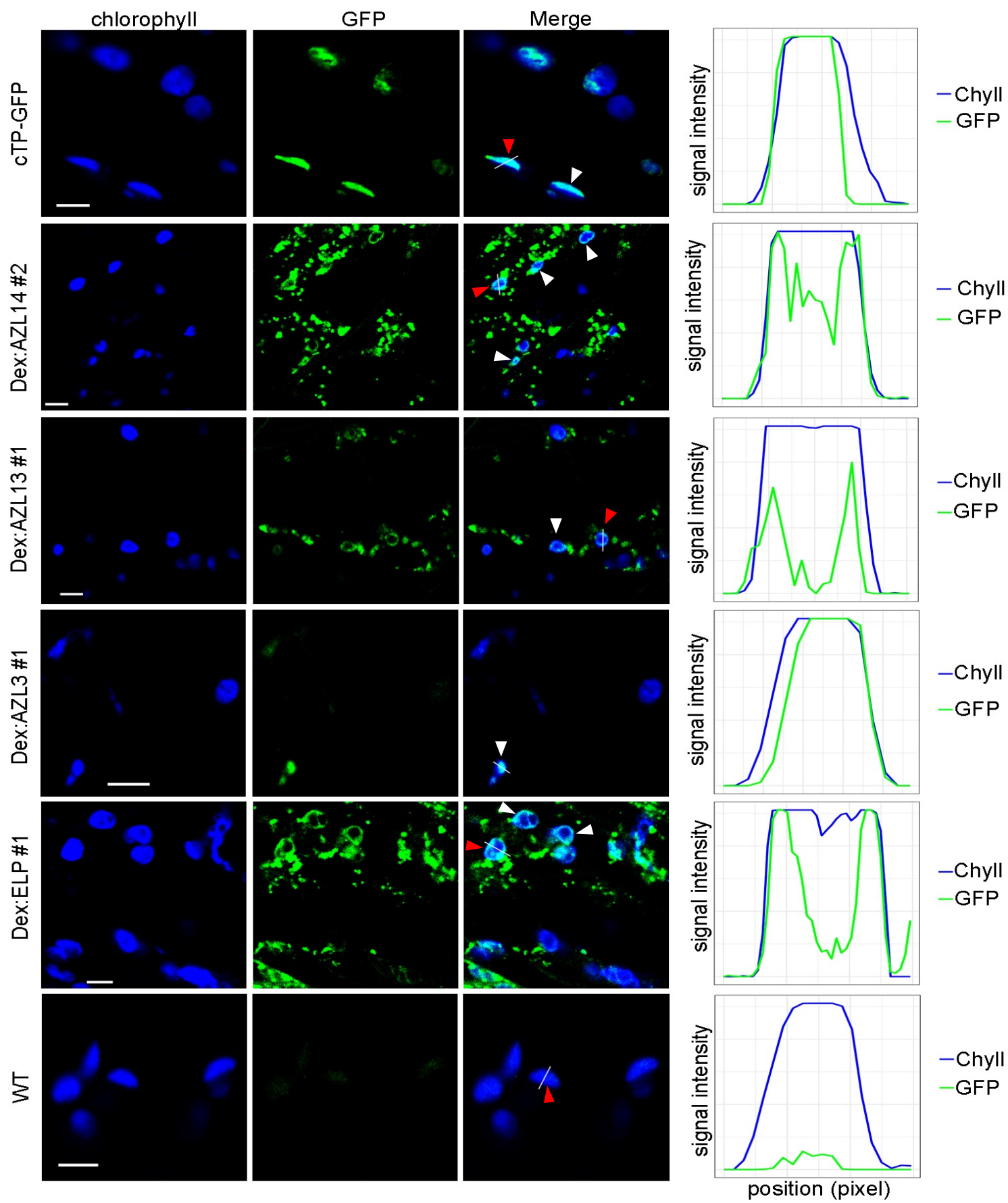

Figure 3. Chloroplast localization of HyPRP:GFP proteins in Arabidopsis transgenic lines. Laser scanning confocal micrographs of epidermal cells showing chloroplast localization of indicated GFP tagged HyPRPs in 9-12 day-old Arabidopsis transgenic seedlings (hypocotyl). Plants were sprayed with $30 \mu \mathrm{M}$ Dex plus $0.04 \%$ Tween $20,21-24 \mathrm{~h}$ prior to imaging. Fluorescence intensity profiles in the merged images show the overlap of GFP (green) and chlorophyll (Chyll, blue) signal intensities along solid white lines (indicated by the red arrowheads). Note the overlap of GFP (green) and chlorophyll (Chyll, blue) intensities along chloroplast peripheries. The plastid-targeted GFP line (cTP-GFP) (Jiang et al., 2021) and untransformed WT (Col-0) serve as positive and negative controls, respectively, for this experiment. The images were analyzed by ImageJ (Fiji). Similar results were observed in two independent experiments. White arrowheads indicate chloroplast localization. Scale bar is $5 \mu \mathrm{m}$. GFP, green; chloroplast autofluorescence, blue. 

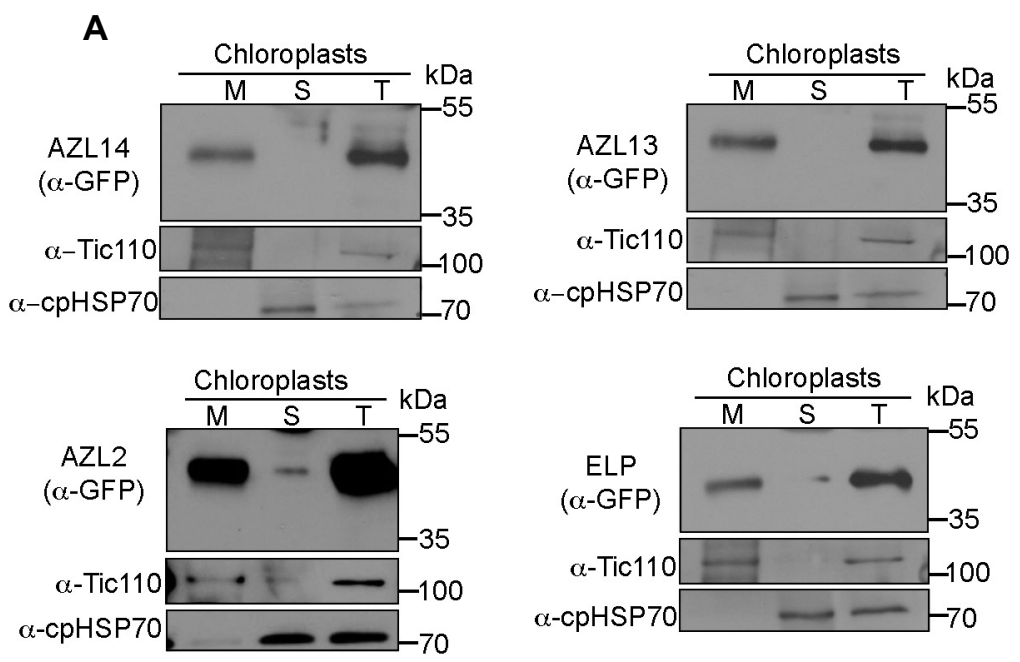

B

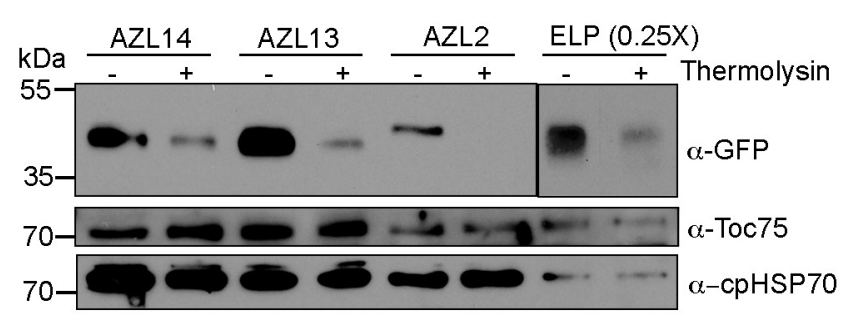

Figure 4. Chloroplast OEM localization of Arabidopsis HyPRPs in $\mathbf{N}$. benthamiana. (A) Immunoblots of total (T), membrane (M) and soluble (S) fractions of isolated chloroplasts from $N$. benthamiana leaves transiently expressing AZL14, AZL13, AZL2 and ELP tagged with GFP at C-terminal under Dex-inducible promoter. HyPRPs were detected with $\alpha$-GFP antibody. $\alpha$-Tic110 and $\alpha$-cpHSP70 antibodies were used to detect the markers of inner envelope membrane, Tic110 and stroma, HSP70. (B) Immunoblots of intact chloroplast fractions expressing HyPRP:GFPs as in (A) treated with buffer (-) or thermolysin (+). The digestion of HyPRP:GFPs by thermolysin was evaluated by $\alpha$-GFP antibody. Due to the higher observed expression of ELP:GFP compared to other HyPRP:GFPs, 1/4X total protein was loaded for this sample. A low exposure for ELP:GFP with $\alpha$-GFP antibody is shown. The antibodies for OEM protein Toc75 and stromal HSP70 were used to detect the non-digestible controls. These experiments were repeated two-three times with similar results. 
A

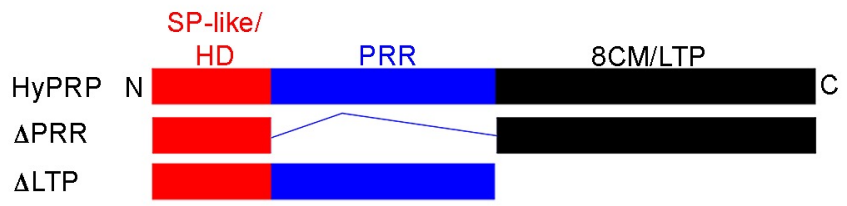

B
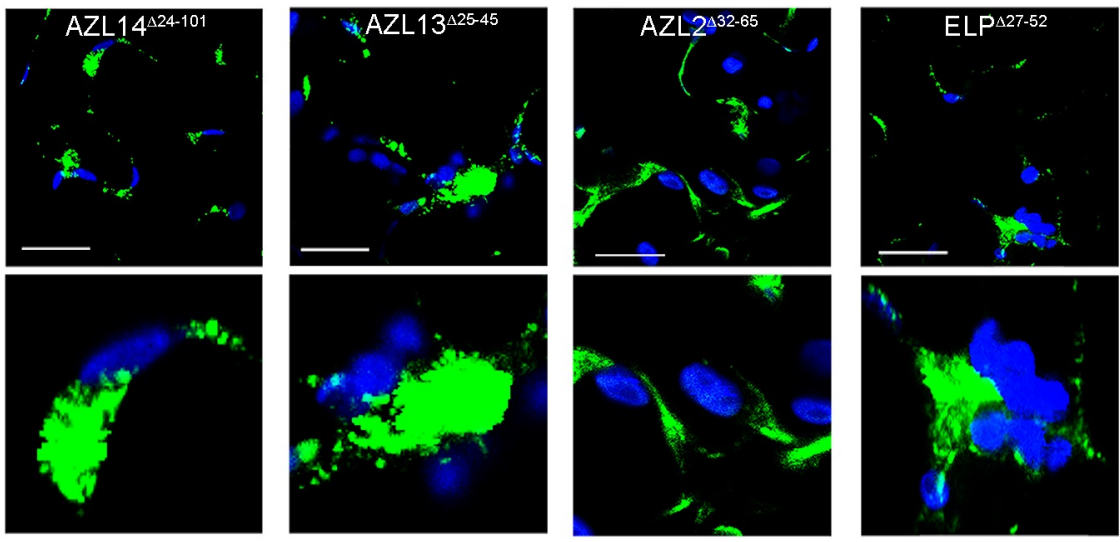

C
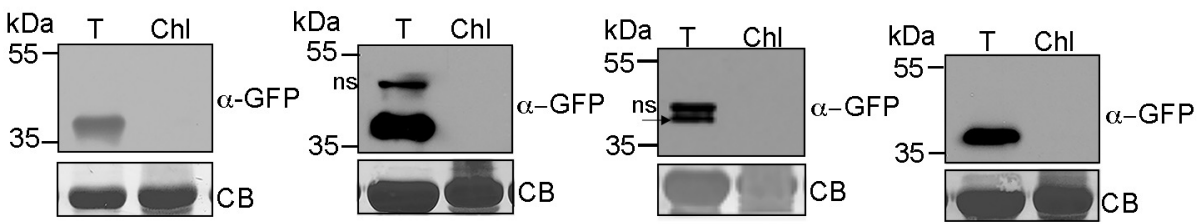

D
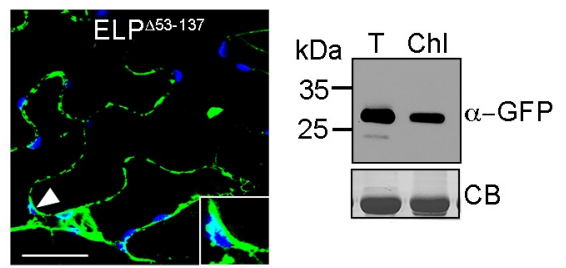

Figure 5. Subcellular localization of the truncated versions of HyPRP:GFPs in $\mathbf{N}$. benthamiana. (A) Schematic of a HyPRP showing N-terminal SP-like or HD, PRR and the C-terminal $8 \mathrm{CM} / \mathrm{LTP}$ domain. The $\triangle \mathrm{PRR}$ and $\triangle \mathrm{LTP}$ versions are also depicted. (B) Confocal micrographs showing the localization of $\triangle P R R$ mutants of GFP-tagged HyPRPs in epidermal cells under Dex-inducible promoter. Lower panels show the enlarged views of chloroplasts. (C) Immunoblots of total ( $T$ ) and chloroplast (Chl) fractions from $N$. benthamiana leaves transiently expressing HyPRP:GFP proteins as in (B). GFP-tagged HyPRPs were detected by $\alpha$-GFP antibody. ns is nonspecific band; arrow indicates specific band. The same blots stained with Coomassie blue (CB) show protein loading. Similar results were observed in two or more independent experiments for AZL14, AZL13 and ELP. For AZL2, the result of one experiment is shown. (D) Confocal micrograph of epidermal cells and immunoblot of total $(\mathrm{T})$ and chloroplast (Chl) fraction showing the subcellular localization of GFPtagged ELP: $\triangle L T P$. Inset shows signal associated with chloroplast. For the micrographs, GFP is green; chloroplast autofluorescence is blue. All Scale bars are $20 \mu \mathrm{m}$. 
A
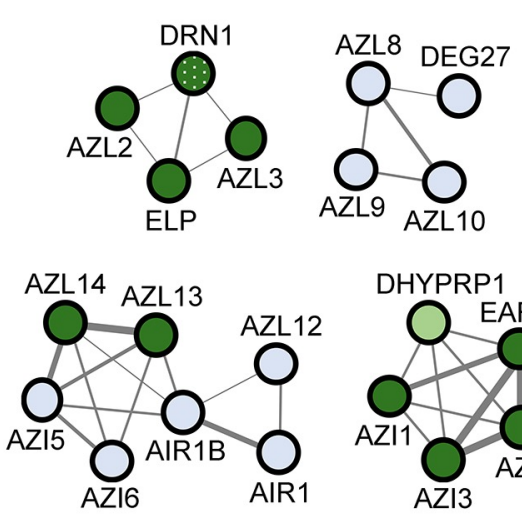

B

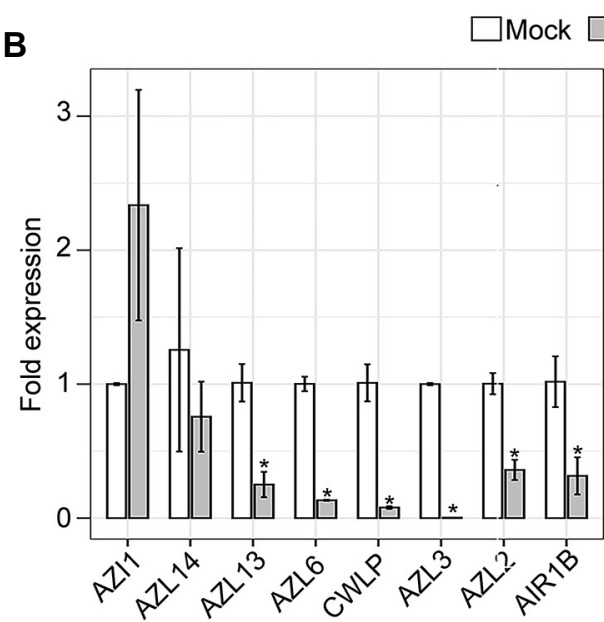

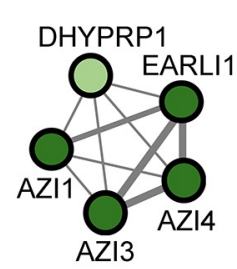
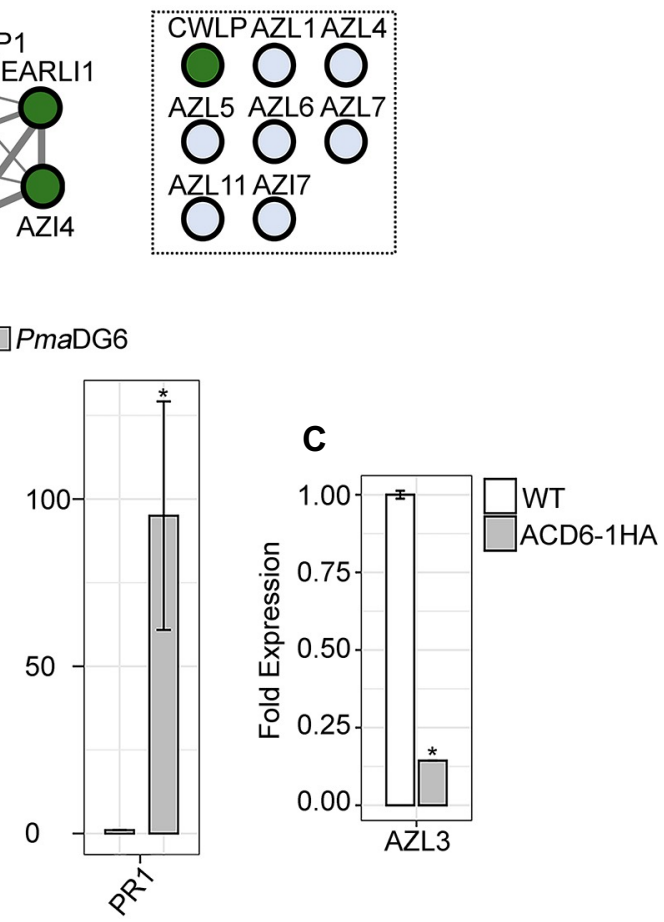

Figure 6. Expression, predicted co-expression and plastid envelope localization of HyPRPs. (A) Network co-expression map of the HyPRP genes using ATTED-II (version 11.0; https://atted.jp/). Solid lines indicate coexpression between the genes included within the top 20 with higher score (Logit Score). Line thickness indicates the co-expression score. Conditionindependent and condition-specific co-expression data is depicted. Green circles indicate HyPRPs targeted to the plastid envelope found in this and our previous (Cecchini et al., 2015) study. Dotted green circle indicates DRN1 plastid targeting evidenced in this and other studies (Peltier et al., 2004; Tomizioli et al., 2014). Light green circles indicate partial targeting to plastids. Inside the dashed box are the genes with no co-expression or a Logit score lower than 3. (B) Transcript levels of HyPRPs in PmaDG6-infected WT (Col-0) leaves 18hpi. PR1 induction shows that the defense response was activated in these plants. (C) Transcript levels of AZL3 in constitutively defense active mutant $A C D 6-1 H A$. In (B and C) EF1 $\alpha$ was used as an internal reference. Error bars indicate SEM from two biological replicates and two technical replicates. ${ }^{*} p<0.05$, student's t-test. 
A

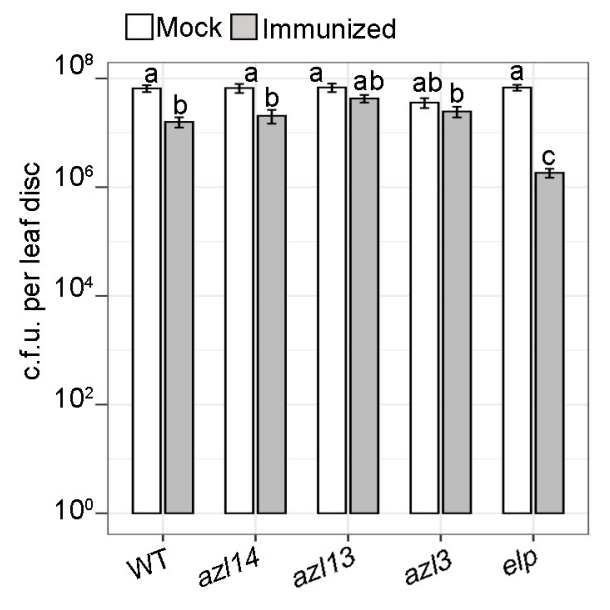

B

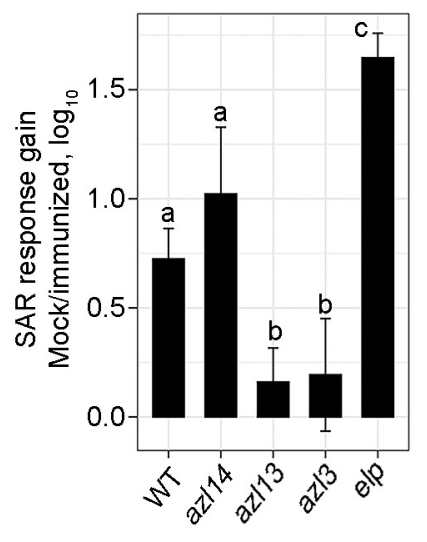

C

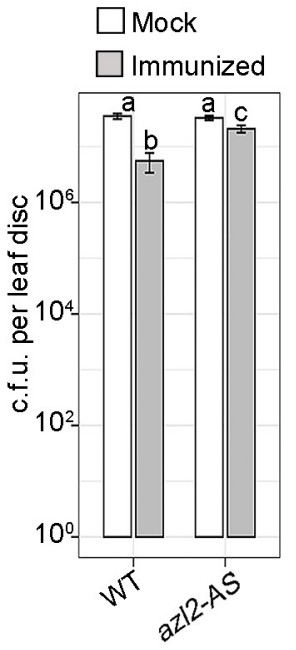

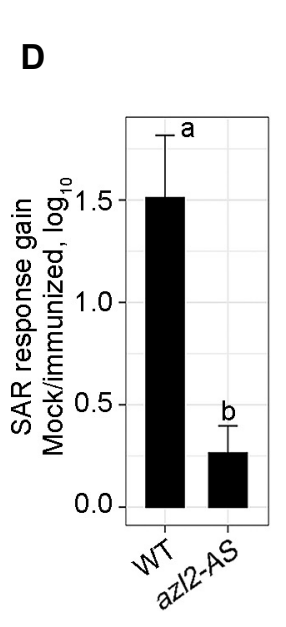

\section{E}
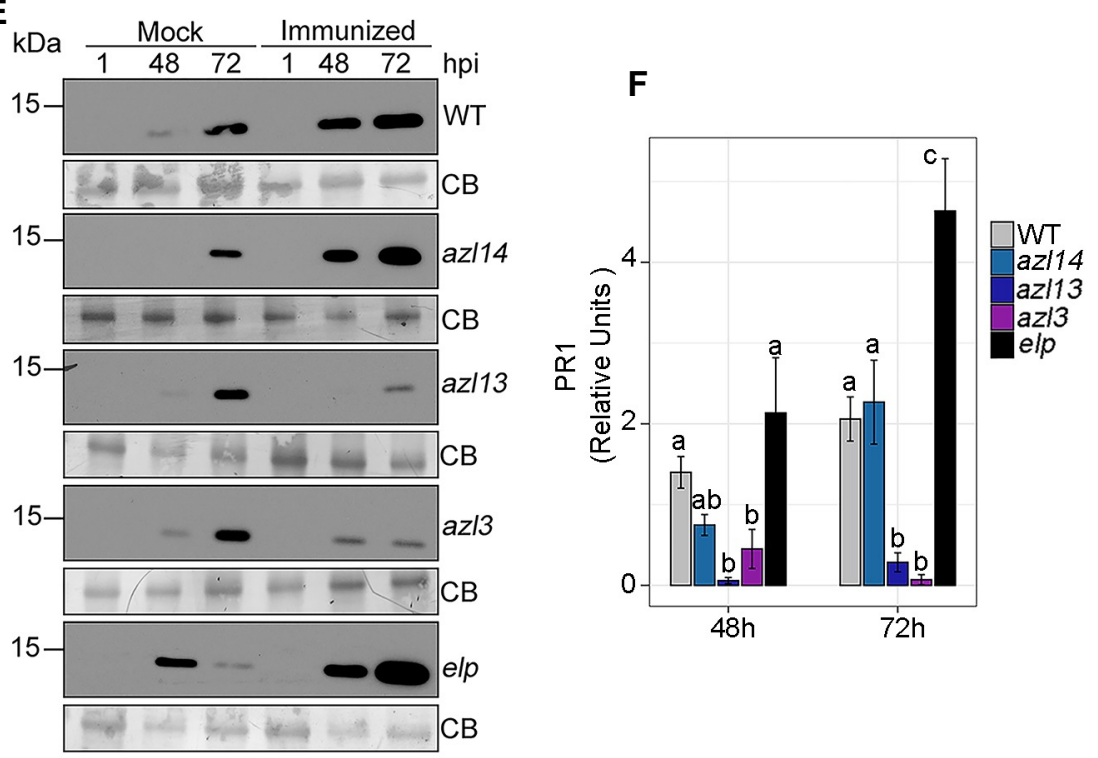

G

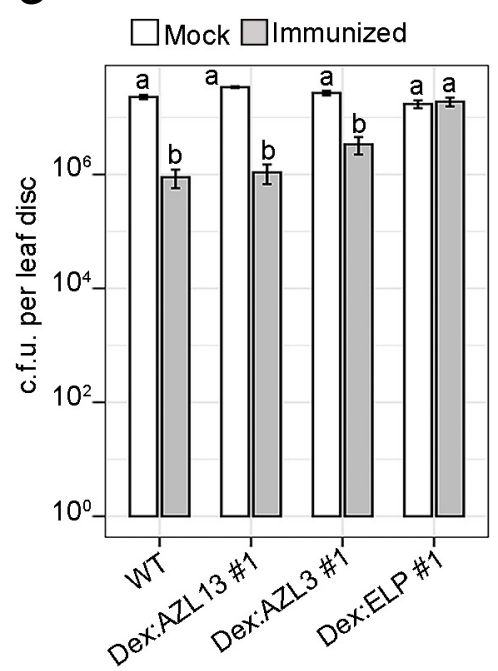

H

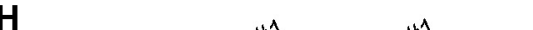

I

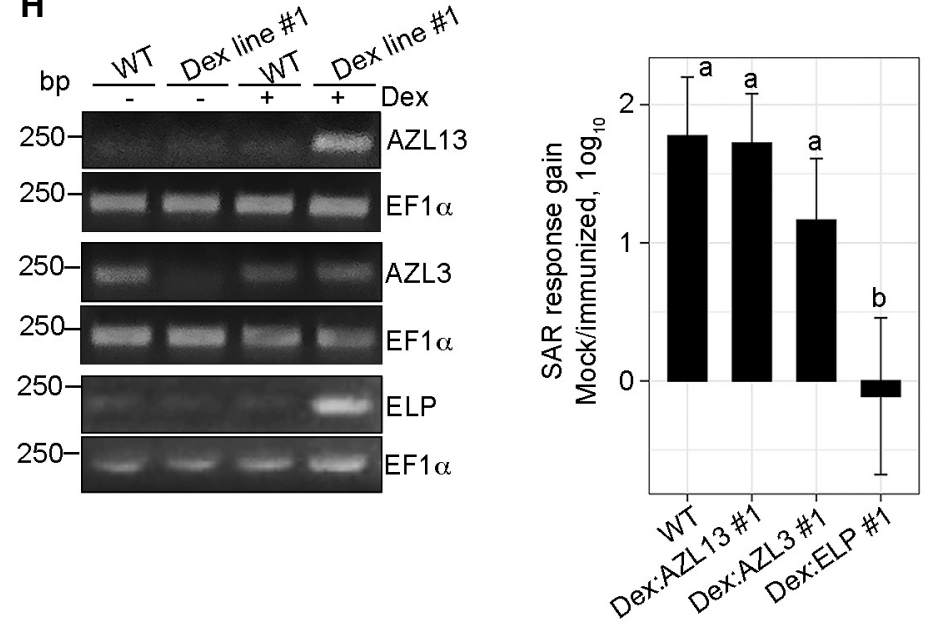


Figure 7. SAR in Arabidopsis HyPRP mutants. (A, C) Growth of virulent bacteria PmaDG3 in systemic leaves of WT (Col-0) and indicated mutants three days after infection. The plants were previously immunized in local leaves with avirulent PmaDG6 or mock treated, 2 days prior to the secondary infection. The mean c.f.u. of two-three independent experiments are plotted. (B) Response gain of SAR due to PmaDG6 immunization in (A). (D) Response gain of SAR due to PmaDG6 immunization in (C). (E) PR1 induction at the indicated time points in the uninfected systemic leaves of plants that were locally mock treated or immunized with PmaDG6. (F) Densitometric quantification of PR1 levels in immunized samples shown in (E). The PR1 levels are relative to the non-specific band in Coomassie blue (CB) stained membrane. The mean from two replicates is shown. Quantification was done by ImageJ (Fiji). (G) Growth of virulent bacteria PmaDG3 in systemic leaves of WT (Col-0) and Dex-inducible complementation lines, three days after infection. The plants were mock treated or immunized as in (A and $\mathbf{C}$ ). $30 \mu \mathrm{M}$ Dex plus $0.04 \%$ Tween 20 was sprayed on local and systemic leaves $21 \mathrm{~h}$ before local and distal infection, respectively. (H) RT-PCR of the indicated HyPRP transcripts in Dex-inducible complementation lines. Leaves were collected $21 \mathrm{~h}$ after treatment with $30 \mu \mathrm{M}$ Dex (+) or no treatment (-). Expression of EF1 $\alpha$ was used as control. (I) Response gain of SAR due to PmaDG6 immunization in (G). In the graphs showing 'response gain', error bars indicate uncertainties in the experiment; in all other graphs error bars show SEM. Different letters above the bars indicate statistically significant difference. Anova, SNK test or Student's test, $p<0.05$ 

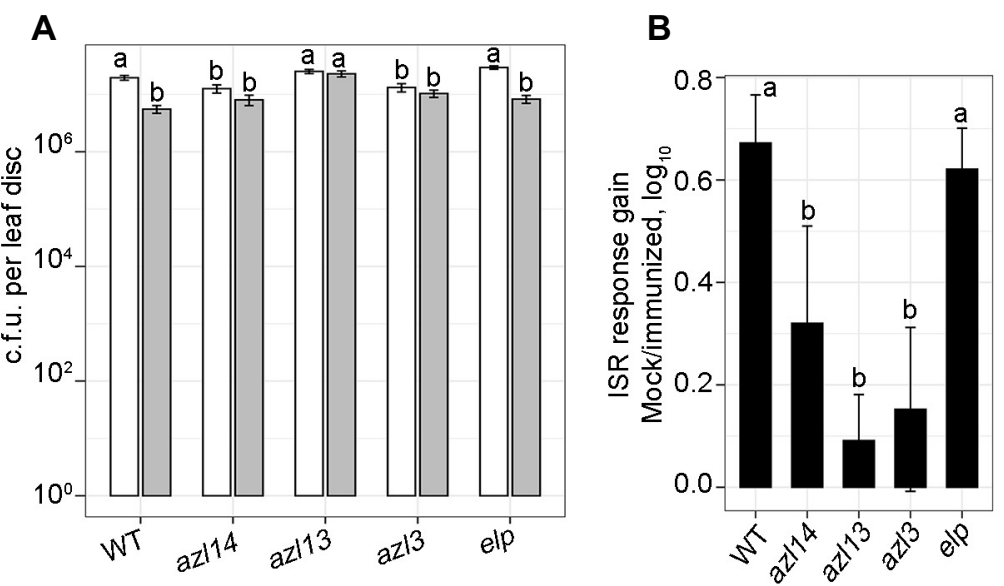

Figure 8. ISR in HyPRPs mutants in Arabidopsis. (A) ISR was assayed by quantification of virulent bacteria PmaDG3 in leaves of plants, three days after infection. The roots of the plants were mock-treated or inoculated with $P$. simiae WCS417, 15-18 days before challenging the leaves with PmaDG3. Error bars show SEM. (B) Response gain of ISR due to $P$. simiae WCS417 immunization in roots of plants in (A). Error bars indicate uncertainties in the experiment. The mean c.f.u. of two independent experiments are plotted. Different letters above the bars indicate statistically significant difference by anova, SNK test, $p<0.05$ 
A
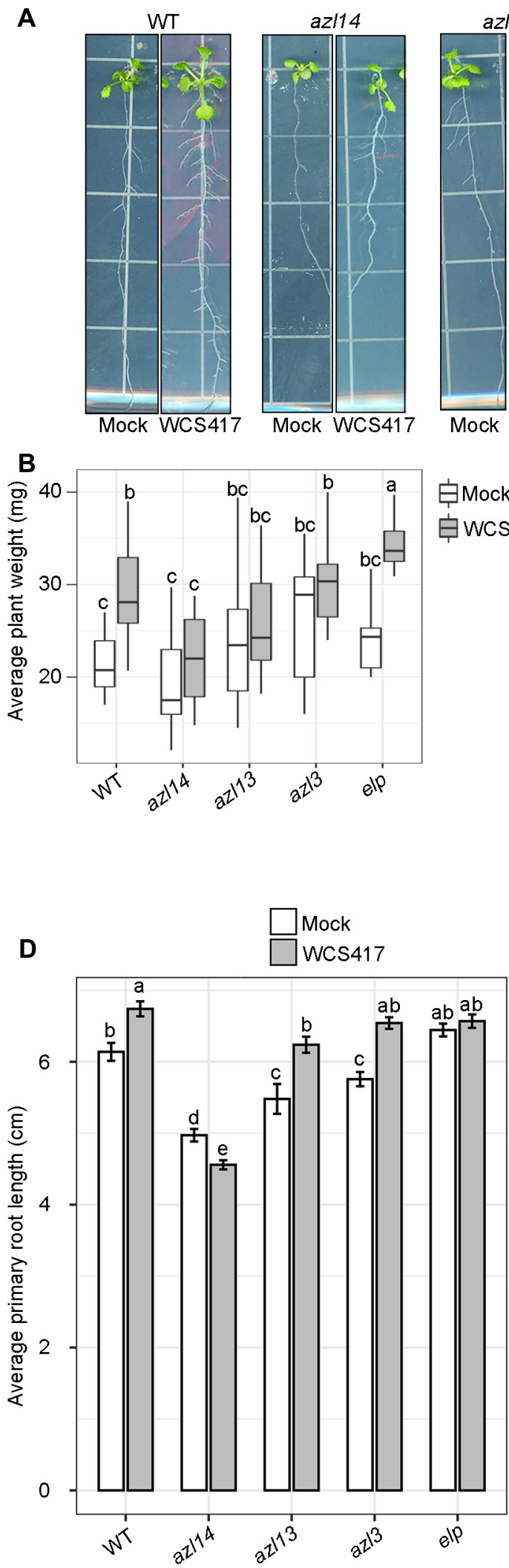
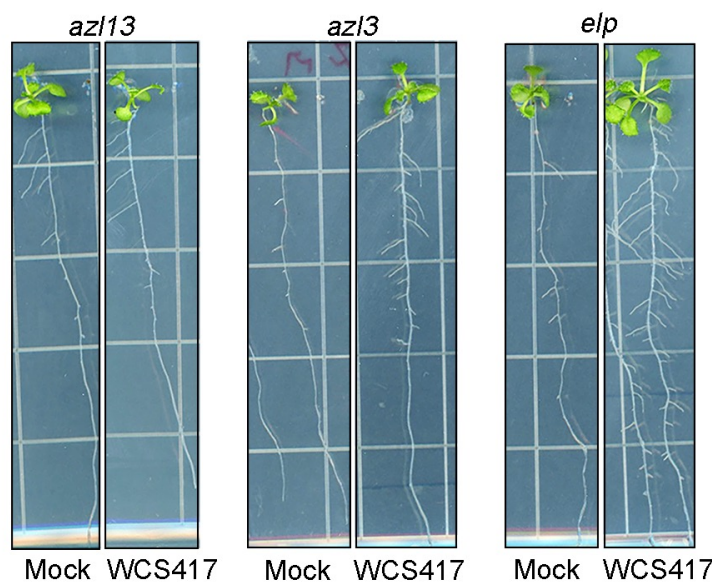

C
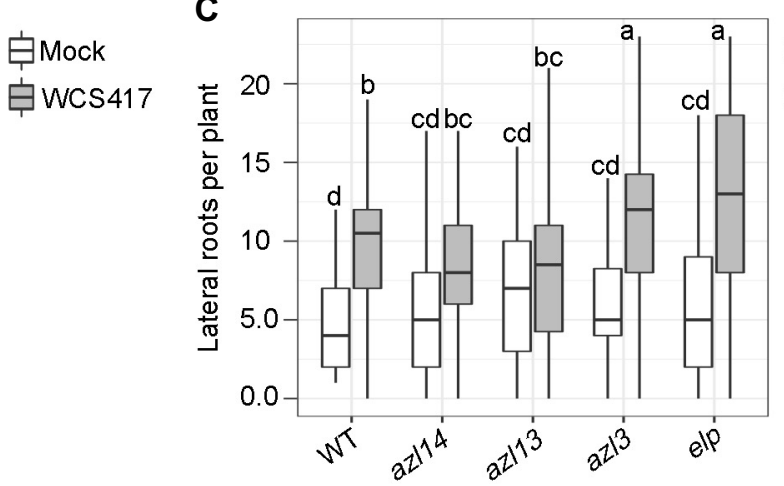

白Mock

追wCS417
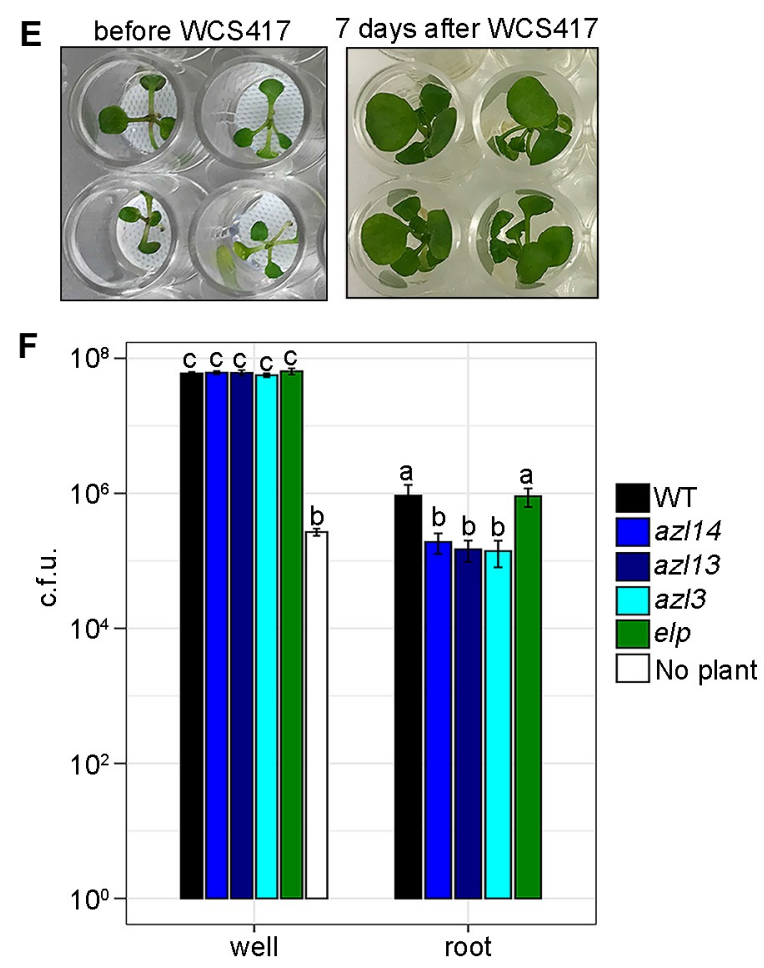
Figure 9. Growth promotion and root-colonization of HyPRP mutants by $P$. simiae WCS417. (A) Representative images of WT (Col-0) and indicated mutants grown on agar plates without sugar and inoculated with mock or $P$. simiae WCS417 (WCS417). 15 day-old seedlings, 10dpi with WCS417 or mock are shown. (B) Fresh weight, (C) number of lateral roots and (D) primary root length in plants inoculated with mock or WCS417 as in (A), n=15-20 plants. Combined data from three independent experiments is plotted. (E) Representative images of WT (Col-0) plants grown hydroponically in 48-well plates before (12 day-old) and 7dpi (19 day-old) with WCS417. (F) Number of c.f.u. of WCS417 in the wells or attached to the roots of WT and mutants. 'No plant' represents the number of c.f.u. of WCS417 in the well-media without any plant present. $\mathrm{n}=20-24$ plants. In all graphs error bars are SEM and different letters above bars indicate statistical differences by anova, SNK test. $p<0.05$. 
A

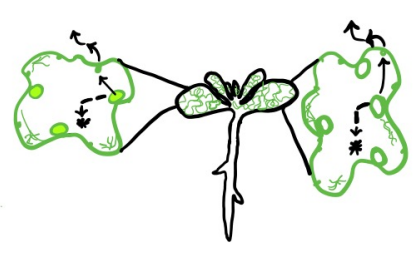

Key

Chloroplast OEM

Chloroplast OEM and/or thylakoid

PM/PD

ER

$\underset{-\rightarrow \text { Signal (long distance) }}{\longrightarrow \text { Signal (local) }}$

B

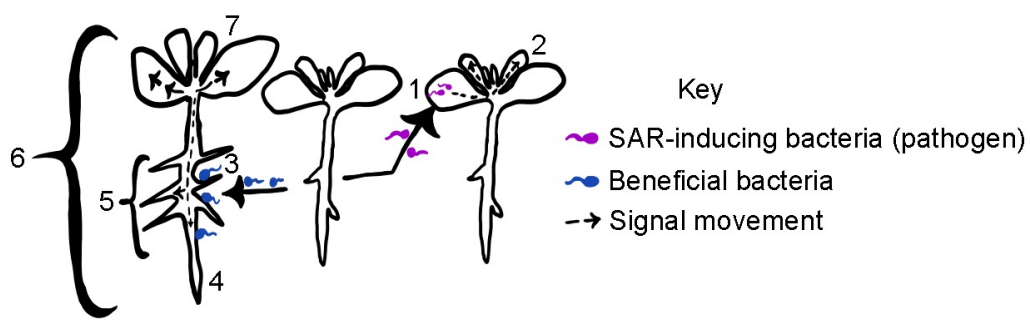

Figure 10. Speculative model and summary of roles of HyPRPs in microbial interactions. (A) Cellular localization and proposed roles of HyPRPs in signal mobilization. Green color indicates organelles and structures in cells where HyPRPs are found. Arrows show the potential mobilization of signals by HyPRPs, either within a cell or locally near the infection (dashed line and ${ }^{*}$ ) or the long-distance mobilization of signals to distal tissue. Although leaf epidermal cells are depicted, similar localization patterns are expected for root cells. HyPRPs with * have roles in local responses to microbes (root or leaf colonization); most also have roles in longer distance responses as well (see part B). Data from this and previous work (Cecchini et al., 2015, 2020; Peltier et al., 2004; Tomizioli et al., 2014) is summarized. The cell on the left shows HyPRPs that likely locate to plastid thylakoids and/or OEMs as well as ER and PM/PD. HyPRPs that fit the left pattern, AZL3* and DRN1*, have positive roles. The cell on the right shows HyPRPs that locate to plastid OEMs as well as ER and/or PM/PDs. HyPRPs that fit the right pattern have positive roles (AZI1, EARLI1, AZL2, AZL13, and AZL14), a mainly negative role (ELP) or have unknown roles (CWLP, DHyPRP, AZI3, AZI4) in microbial responses. (B) Schematic of the steps at the whole plant level that are affected by one or more HyPRPs: 1, leaf colonization by pathogen (DRN1, positive role); 2, increased immunity to distal infections after an immunizing infection at site 1 (SAR: AZI1, EARLI1, AZL2, AZL3, AZL13, positive roles; ELP, negative role); 3, growth of beneficial bacteria in association with roots (AZL3, AZL13, AZL14, positive roles); 4, stimulation of primary root growth in response to beneficial bacteria colonization of the root (ELP, AZL14, positive roles); 5 , stimulation of lateral roots in response to beneficial bacteria colonization of the root (AZL13, AZL14, positive roles; ELP, negative role); 6 , stimulation of whole plant growth by root colonization by beneficial bacteria (AZL3, AZL13, AZL14, positive roles; ELP, negative role); 7, increased immunity of aerial tissue (ISR after root colonization by beneficial bacteria: AZI1, EARLI1, AZL3, AZL13, AZL14, positive roles). 


\section{Parsed Citations}

Albert, I., Hua, C., Nürnberger, T., Pruitt, R.N., and Zhang, L. (2020). Surface Sensor Systems in Plant Immunity. Plant Physiol 182: $1582-$ 1596.

Google Scholar: Author Only Title Only Author and Title

Andersson, M.X., Goksör, M., and Sandelius, AS. (2007). Membrane contact sites: physical attachment between chloroplasts and endoplasmic reticulum revealed by optical manipulation. Plant Signal Behav 2: 185-187.

Google Scholar: Author Only Title Only Author and Title

Ascencio-lbáñez, J.T., Sozzani, R., Lee, T.-J., Chu, T.-M., Wolfinger, R.D., Cella, R., and Hanley-Bowdoin, L. (2008). Global Analysis of Arabidopsis Gene Expression Uncovers a Complex Array of Changes Impacting Pathogen Response and Cell Cycle during

Geminivirus Infection. Plant Physiol. 148: 436-454.

Google Scholar: Author Only Title Only Author and Title

Baum, S., Reimer-Michalski, E.-M., Bolger, A, Mantai, AJ., Benes, V., Usadel, B., and Conrath, U. (2019). Isolation of Open Chromatin Identifies Regulators of Systemic Acquired Resistance. Plant Physiol. 181: 817-833.

Google Scholar: Author Only Title Only Author and Title

Beckers, G.J.M. and Conrath, U. (2007). Priming for stress resistance: from the lab to the field. Curr Opin Plant Biol 10: 425-431.

Google Scholar: Author Only Title Only Author and Title

Beckers, G.J.M., Jaskiewicz, M., Liu, Y., Underwood, W.R., He, S.Y., Zhang, S., and Conrath, U. (2009). Mitogen-activated protein kinases 3 and 6 are required for full priming of stress responses in Arabidopsis thaliana. Plant Cell 21: 944-953.

Google Scholar: Author Only Title Only Author and Title

Berendsen, R.L., Pieterse, C.M.J., and Bakker, P.AH.M. (2012). The rhizosphere microbiome and plant health. Trends Plant Sci 17: 478-486.

Google Scholar: Author Only Title Only Author and Title

Bobik, K. and Burch-Smith, T.M. (2015). Chloroplast signaling within, between and beyond cells. Front Plant Sci 6: 781.

Google Scholar: Author Only Title Only Author and Title

Bonnot, T., Gillard, M., and Nagel, D. (2019). A Simple Protocol for Informative Visualization of Enriched Gene Ontology Terms. BIOPROTOCOL 9.

Google Scholar: Author Only Title Only Author and Title

Bruce, B.D. (2001). The paradox of plastid transit peptides: conservation of function despite divergence in primary structure.

Biochimica et Biophysica Acta (BBA) - Molecular Cell Research 1541: 2-21.

Google Scholar: Author Only Title Only Author and Title

Bulgarelli, D., Schlaeppi, K., Spaepen, S., Ver Loren van Themaat, E., and Schulze-Lefert, P. (2013). Structure and functions of the bacterial microbiota of plants. Annu Rev Plant Biol 64: 807-838.

Google Scholar: Author Only Title Only Author and Title

Caplan, J.L., Kumar, AS., Park, E., Padmanabhan, M.S., Hoban, K., Modla, S., Czymmek, K., and Dinesh-Kumar, S.P. (2015). Chloroplast Stromules Function during Innate Immunity. Dev Cell 34: 45-57.

Google Scholar: Author Only Title Only Author and Title

Carella, P. (2020). Xylem-Mobile Oxylipins Are Critical Regulators of Induced Systemic Resistance in Maize. Plant Cell 32: 13-14.

Google Scholar: Author Only Title Only Author and Title

Cecchini, N., Song, Y., Roychoudhry, S., Greenberg, J., and Haney, C. (2019a). An Improved Bioassay to Study Arabidopsis Induced Systemic Resistance (ISR) Against Bacterial Pathogens and Insect Pests. BIO-PROTOCOL 9.

Google Scholar: Author Only Title Only Author and Title

Cecchini, N.M., Roychoudhry, S., Speed, D.J., Steffes, K., Tambe, A, Zodrow, K., Konstantinoff, K., Jung, H.W., Engle, N.L., Tschaplinski, T.J., and Greenberg, J.T. (2019b). Underground Azelaic Acid-Conferred Resistance to Pseudomonas syringae in Arabidopsis. MPMI 32: 86-94.

Google Scholar: Author Only Title Only Author and Title

Cecchini, N.M., Speed, D.J., Roychoudhry, S., and Greenberg, J.T. (2020). Kinases and protein motifs required for AZ1 plastid localization and trafficking during plant defense induction. Plant $\mathrm{J}$.

Google Scholar: Author Only Title Only Author and Title

Cecchini, N.M., Steffes, K., Schläppi, M.R., Gifford, AN., and Greenberg, J.T. (2015). Arabidopsis AZ1 family proteins mediate signal mobilization for systemic defence priming. Nat Commun 6: 7658.

Google Scholar: Author Only Title Only Author and Title

Chen, Y.-L., Chen, L.-J., and Li, H.-M. (2016). Polypeptide Transport-Associated Domains of the Toc75 Channel Protein Are Located in the Intermembrane Space of Chloroplasts. Plant Physiol 172: 235-243.

Google Scholar: Author Only Title Only Author and Title 
Clough, S.J. and Bent, AF. (1998). Floral dip: a simplified method for Agrobacterium-mediated transformation of Arabidopsis thaliana.

Plant J 16: 735-743.

Google Scholar: Author Only Title Only Author and Title

Dempsey, D.A, Vot, AC., Wildermuth, M.C., and Klessig, D.F. (2011). Salicylic Acid biosynthesis and metabolism Arabidopsis Book 9: e0156.

Google Scholar: Author Only Title Only Author and Title

Dhar, N., Caruana, J., Erdem, I., and Raina, R. (2020). An Arabidopsis DISEASE RELATED NONSPECIFIC LIPID TRANSFER PROTEIN 1 is required for resistance against various phytopathogens and tolerance to salt stress. Gene: 144802.

Google Scholar: Author Only Title Only Author and Title

Efthimiadou, A, Katsenios, N., Chanioti, S., Giannoglou, M., Djordjevic, N., and Katsaros, G. (2020). Effect of foliar and soil application of plant growth promoting bacteria on growth, physiology, yield and seed quality of maize under Mediterranean conditions. Sci Rep 10: 21060.

Google Scholar: Author Only Title Only Author and Title

Emanuelsson, O., Nielsen, H., Brunak, S., and von Heijne, G. (2000). Predicting Subcellular Localization of Proteins Based on their Nterminal Amino Acid Sequence. Journal of Molecular Biology 300: 1005-1016.

Google Scholar: Author Only Title Only Author and Title

Emanuelsson, O., Nielsen, H., and Heijne, G.V. (1999). ChloroP, a neural network-based method for predicting chloroplast transit peptides and their cleavage sites. Protein Sci. 8: 978-984.

Google Scholar: Author Only Title Only Author and Title

Fu, ZQ. and Dong, X. (2013). Systemic acquired resistance: turning local infection into global defense. Annu Rev Plant Biol 64: $839-863$. Google Scholar: Author Only Title Only Author and Title

Gupta, A and Senthil-Kumar, M. (2017). Transcriptome changes in Arabidopsis thaliana infected with Pseudomonas syringae during drought recovery. Sci Rep 7: 9124.

Google Scholar: Author Only Title Only Author and Title

Haney, C.H., Samuel, B.S., Bush, J., and Ausubel, F.M. (2015). Associations with rhizosphere bacteria can confer an adaptive advantage to plants. Nature Plants 1: 15051.

Google Scholar: Author Only Title Only Author and Title

Hartmann, M., Kim, D., Bernsdorff, F., Ajami-Rashidi, Z, Scholten, N., Schreiber, S., Zeier, T., Schuck, S., Reichel-Deland, V., and Zeier, J. (2017). Biochemical Principles and Functional Aspects of Pipecolic Acid Biosynthesis in Plant Immunity. Plant Physiol. 174: 124-153.

Google Scholar: Author Only Title Only Author and Title

Helle, S.C.J., Kanfer, G., Kolar, K., Lang, A, Michel, AH., and Kornmann, B. (2013). Organization and function of membrane contact sites. Biochim Biophys Acta 1833: 2526-2541.

Google Scholar: Author Only Title Only Author and Title

Hofmann, N.R. and Theg, S.M. (2005). Chloroplast outer membrane protein targeting and insertion. Trends Plant Sci $10: 450-457$.

Google Scholar: Author Only Title Only Author and Title

Howard, B.E., Hu, Q., Babaoglu, AC., Chandra, M., Borghi, M., Tan, X., He, L., Winter-Sederoff, H., Gassmann, W., Veronese, P., and Heber, S. (2013). High-throughput RNA sequencing of pseudomonas-infected Arabidopsis reveals hidden transcriptome complexity and novel splice variants. PLoS One 8: e74183.

Google Scholar: Author Only Title Only Author and Title

Hruz, T., Laule, O., Szabo, G., Wessendorp, F., Bleuler, S., Oertle, L., Widmayer, P., Gruissem, W., and Zmmermann, P. (2008).

Genevestigator v3: a reference expression database for the meta-analysis of transcriptomes. Adv Bioinformatics 2008: 420747.

Google Scholar: Author Only Title Only Author and Title

Jaskiewicz, M., Conrath, U., and Peterhänsel, C. (2011). Chromatin modification acts as a memory for systemic acquired resistance in the plant stress response. EMBO Rep 12: 50-55.

Google Scholar: Author Only Title Only Author and Title

Jayasinghe, S., Hristova, K., and White, S.H. (2001). Energetics, stability, and prediction of transmembrane helices11Edited by G. von Heijne. Journal of Molecular Biology 312: 927-934.

Google Scholar: Author Only Title Only Author and Title

Jiang, S.-C., Engle, N.L., Banday, ZZ, Cecchini, N.M., Jung, H.W., Tschaplinski, T.J., and Greenberg, J.T. (2021). ALD1 accumulation in Arabidopsis epidermal plastids confers local and nonautonomous disease resistance. J Exp Bot.

Google Scholar: Author Only Title Only Author and Title

Jülke, S. and Ludwig-Müller, J. (2015). Response of Arabidopsis thaliana Roots with Altered Lipid Transfer Protein (LTP) Gene

Expression to the Clubroot Disease and Salt Stress. Plants 5: 2.

Google Scholar: Author Only Title Only Author and Title

Jung, H.W., Tschaplinski, T.J., Wang, L., Glazebrook, J., and Greenberg, J.T. (2009). Priming in Systemic Plant Immunity. Science 324: 89-91. 
Kachroo, A and Kachroo, P. (2020). Mobile signals in systemic acquired resistance. Curr Opin Plant Biol 58: 41-47.

Google Scholar: Author Only Title Only Author and Title

Kleffmann, T., Russenberger, D., von Zychlinski, A, Christopher, W., Sjölander, K., Gruissem, W., and Baginsky, S. (2004). The Arabidopsis thaliana Chloroplast Proteome Reveals Pathway Abundance and Novel Protein Functions. Current Biology 14: 354-362.

Google Scholar: Author Only Title Only Author and Title

Küchler, M., Decker, S., Hörmann, F., Soll, J., and Heins, L. (2002). Protein import into chloroplasts involves redox-regulated proteins. EMBO J 21: 6136-6145.

Google Scholar: Author Only Title Only Author and Title

Lee, D.W., Kim, J.K., Lee, S., Choi, S., Kim, S., and Hwang, I. (2008). Arabidopsis Nuclear-Encoded Plastid Transit Peptides Contain Multiple Sequence Subgroups with Distinctive Chloroplast-Targeting Sequence Motifs. Plant Cell 20: 1603-1622.

Google Scholar: Author Only Title Only Author and Title

Lee, D.W., Yoo, Y.-J., Razzak, Md.A, and Hwang, I. (2018). Prolines in Transit Peptides Are Crucial for Efficient Preprotein

Translocation into Chloroplasts. Plant Physiol. 176: 663-677.

Google Scholar: Author Only Title Only Author and Title

Lee, J., Lee, H., Kim, J., Lee, S., Kim, D.H., Kim, S., and Hwang, I. (2011). Both the Hydrophobicity and a Positively Charged Region Flanking the C-Terminal Region of the Transmembrane Domain of Signal-Anchored Proteins Play Critical Roles in Determining Their Targeting Specificity to the Endoplasmic Reticulum or Endosymbiotic Organelles in Arabidopsis Cells. Plant Cell 23: 1588-1607. Google Scholar: Author Only Title Only Author and Title

Lee, J.-Y. (2015). Plasmodesmata: a signaling hub at the cellular boundary. Curr Opin Plant Biol 27: 133-140.

Google Scholar: Author Only Title Only Author and Title

Li, B.-C., Zhang, C., Chai, Q.-X., Han, Y.-Y., Wang, X.-Y., Liu, M.-X., Feng, H., and Xu, Z-Q. (2014). Plasmalemma localisation of DOUBLE HYBRID PROLINE-RICH PROTEIN 1 and its function in systemic acquired resistance of Arabidopsis thaliana. Functional Plant Biol. 41: 768.

Google Scholar: Author Only Title Only Author and Title

Li, T., Xiao, Z, Li, H., Liu, C., Shen, W., and Gao, C. (2020). A Combinatorial Reporter Set to Visualize the Membrane Contact Sites Between Endoplasmic Reticulum and Other Organelles in Plant Cell. Front. Plant Sci. 11: 1280.

Google Scholar: Author Only Title Only Author and Title

Lim, G.-H., Shine, M.B., de Lorenzo, L., Yu, K., Cui, W., Navarre, D., Hunt, AG., Lee, J.-Y., Kachroo, A, and Kachroo, P. (2016).

Plasmodesmata Localizing Proteins Regulate Transport and Signaling during Systemic Acquired Immunity in Plants. Cell Host Microbe 19: 541-549.

Google Scholar: Author Only Title Only Author and Title

Lu, H., Liu, Y., and Greenberg, J.T. (2005). Structure-function analysis of the plasma membrane- localized Arabidopsis defense component ACD6. Plant J 44: 798-809.

Google Scholar: Author Only Title Only Author and Title

Mészáros, T., Helfer, A, Hatzimasoura, E., Magyar, Z, Serazetdinova, L., Rios, G., Bardóczy, V., Teige, M., Koncz, C., Peck, S., and Bögre, L. (2006). The Arabidopsis MAP kinase kinase MKK1 participates in defence responses to the bacterial elicitor flagellin. The Plant Journal 48: 485-498.

Google Scholar: Author Only Title Only Author and Title

Mine, A, Seyfferth, C., Kracher, B., Berens, M.L., Becker, D., and Tsuda, K. (2018). The Defense Phytohormone Signaling Network Enables Rapid, High-Amplitude Transcriptional Reprogramming during Effector-Triggered Immunity. Plant Cell 30: 1199-1219.

Google Scholar: Author Only Title Only Author and Title

Mittler, R., Vanderauwera, S., Gollery, M., and Van Breusegem, F. (2004). Reactive oxygen gene network of plants. Trends in Plant Science 9: 490-498.

Google Scholar: Author Only Title Only Author and Title

Mohr, P.G. and Cahill, D.M. (2007). Suppression by ABA of salicylic acid and lignin accumulation and the expression of multiple genes, in Arabidopsis infected with Pseudomonas syringae pv. tomato. Funct Integr Genomics 7: 181-191.

Google Scholar: Author Only Title Only Author and Title

Nielsen, H., Engelbrecht, J., Brunak, S., and von Heijne, G. (1997). Identification of prokaryotic and eukaryotic signal peptides and prediction of their cleavage sites. Protein Eng 10: 1-6.

Google Scholar: Author Only Title Only Author and Title

Peltier, J.-B., Ytterberg, AJ., Sun, Q., and van Wijk, K.J. (2004). New functions of the thylakoid membrane proteome of Arabidopsis thaliana revealed by a simple, fast, and versatile fractionation strategy. J Biol Chem 279: 49367-49383.

Google Scholar: Author Only Title Only Author and Title

Pieterse, C.M., van Wees, S.C., Hoffland, E., van Pelt, J.A, and van Loon, L.C. (1996). Systemic resistance in Arabidopsis induced by 
biocontrol bacteria is independent of salicylic acid accumulation and pathogenesis-related gene expression. Plant Cell 8: $1225-1237$. Google Scholar: Author Only Title Only Author and Title

Pieterse, C.M., van Wees, S.C., van Pelt, J.A, Knoester, M., Laan, R., Gerrits, H., Weisbeek, P.J., and van Loon, L.C. (1998). Anovel signaling pathway controlling induced systemic resistance in Arabidopsis. Plant Cell 10: 1571-1580.

Google Scholar: Author Only Title Only Author and Title

Pieterse, C.M.J., Zamioudis, C., Berendsen, R.L., Weller, D.M., Van Wees, S.C.M., and Bakker, P.AH.M. (2014). Induced systemic resistance by beneficial microbes. Annu Rev Phytopathol 52: 347-375.

Google Scholar: Author Only Title Only Author and Title

Pitzschke, A, Datta, S., and Persak, H. (2014). Salt stress in Arabidopsis: lipid transfer protein AZ1 and its control by mitogen-activated protein kinase MPK3. Mol Plant 7: 722-738.

Google Scholar: Author Only Title Only Author and Title

Qutob, D. et al. (2006). Phytotoxicity and Innate Immune Responses Induced by Nep1-Like Proteins. Plant Cell 18: 3721-3744.

Google Scholar: Author Only Title Only Author and Title

Rekhter, D., Lüdke, D., Ding, Y., Feussner, K., Zenkiewicz, K., Lipka, V., Wiermer, M., Zhang, Y., and Feussner, I. (2019). Isochorismatederived biosynthesis of the plant stress hormone salicylic acid. Science 365: 498-502.

Google Scholar: Author Only Title Only Author and Title

Seguel, A, Jelenska, J., Herrera-Vásquez, A, Marr, S.K., Joyce, M.B., Gagesch, K.R., Shakoor, N., Jiang, S.-C., Fonseca, A, Wildermuth, M.C., Greenberg, J.T., and Holuigue, L. (2018). PROHIBITIN3 Forms Complexes with ISOCHORISMATE SYNTHASE1 to Regulate

Stress-Induced Salicylic Acid Biosynthesis in Arabidopsis. Plant Physiol 176: 2515-2531.

Google Scholar: Author Only Title Only Author and Title

Serrano, M., Wang, B., Aryal, B., Garcion, C., Abou-Mansour, E., Heck, S., Geisler, M., Mauch, F., Nawrath, C., and Métraux, J.-P. (2013). Export of Salicylic Acid from the Chloroplast Requires the Multidrug and Toxin Extrusion-Like Transporter EDS5. Plant Physiol. 162: 1815-1821.

Google Scholar: Author Only Title Only Author and Title

Siemens, J., Keller, I., Sarx, J., Kunz, S., Schuller, A, Nagel, W., Schmülling, T., Parniske, M., and Ludwig-Müller, J. (2006). Transcriptome Analysis of Arabidopsis Clubroots Indicate a Key Role for Cytokinins in Disease Development. MPMI 19 : $480-494$.

Google Scholar: Author Only Title Only Author and Title

Snider, C., Jayasinghe, S., Hristova, K., and White, S.H. (2009). MPEx: Atool for exploring membrane proteins. Protein Science 18: 2624-2628.

Google Scholar: Author Only Title Only Author and Title

Stringlis, I.A, Proietti, S., Hickman, R., Van Verk, M.C., Zamioudis, C., and Pieterse, C.M.J. (2018). Root transcriptional dynamics induced by beneficial rhizobacteria and microbial immune elicitors reveal signatures of adaptation to mutualists. Plant $\mathrm{J} 93: 166-180$.

Google Scholar: Author Only Title Only Author and Title

Thilmony, R., Underwood, W., and He, S.Y. (2006). Genome-wide transcriptional analysis of the Arabidopsis thaliana interaction with the plant pathogen Pseudomonas syringae pv. tomato DC3000 and the human pathogen Escherichia coli O157:H7. The Plant Journal 46:

34-53.

Google Scholar: Author Only Title Only Author and Title

Tomizioli, M. et al. (2014). Deciphering Thylakoid Sub-compartments using a Mass Spectrometry-based Approach. Mol Cell Proteomics 13: 2147-2167.

Google Scholar: Author Only Title Only Author and Title

Toulmay, A and Prinz, W.A (2011). Lipid transfer and signaling at organelle contact sites: the tip of the iceberg. Curr Opin Cell Biol 23: 458-463.

Google Scholar: Author Only Title Only Author and Title

Tripp, J., Inoue, K., Keegstra, K., and Froehlich, J.E. (2007). A novel serine/proline-rich domain in combination with a transmembrane domain is required for the insertion of AtTic40 into the inner envelope membrane of chloroplasts. Plant J. 52: $824-838$.

Google Scholar: Author Only Title Only Author and Title

Wintermans, P.C.A, Bakker, P.AH.M., and Pieterse, C.M.J. (2016). Natural genetic variation in Arabidopsis for responsiveness to plant growth-promoting rhizobacteria. Plant Mol Biol 90: 623-634.

Google Scholar: Author Only Title Only Author and Title

Yu, K., Soares, J.M., Mandal, M.K., Wang, C., Chanda, B., Gifford, AN., Fowler, J.S., Navarre, D., Kachroo, A, and Kachroo, P. (2013). A Feedback Regulatory Loop between G3P and Lipid Transfer Proteins DIR1 and AZ1 Mediates Azelaic-Acid-Induced Systemic Immunity. Cell Reports 3: 1266-1278.

Google Scholar: Author Only Title Only Author and Title

Yu, Z et al. (2020). The Brassicaceae-specific secreted peptides, STMPs, function in plant growth and pathogen defense. J Integr Plant Biol 62: 403-420.

Google Scholar: Author Only Title Only Author and Title 
bioRxiv preprint doi: https://doi.org/10.1101/2021.08.26.457806; this version posted August 27, 2021. The copyright holder for this preprint (which was not certified by peer review) is the author/funder, who has granted bioRxiv a license to display the preprint in perpetuity. It is made available under aCC-BY-NC 4.0 International license.

Zamioudis, C., Hanson, J., and Pieterse, C.M.J. (2014). $\beta$-Glucosidase BGLU42 is a MYB72-dependent key regulator of rhizobacteriainduced systemic resistance and modulates iron deficiency responses in Arabidopsis roots. New Phytol 204: $368-379$.

Google Scholar: Author Only Title Only Author and Title

Zamioudis, C., Mastranesti, P., Dhonukshe, P., Blilou, I., and Pieterse, C.M.J. (2013). Unraveling root developmental programs initiated by beneficial Pseudomonas spp. bacteria. Plant Physiol 162: 304-318.

Google Scholar: Author Only Title Only Author and Title

Zpfel, C. (2008). Pattern-recognition receptors in plant innate immunity. Curr. Opin. Immunol. 20: 10-16.

Google Scholar: Author Only Title Only Author and Title

Zoeller, M., Stingl, N., Krischke, M., Fekete, A, Waller, F., Berger, S., and Mueller, M.J. (2012). Lipid Profiling of the Arabidopsis Hypersensitive Response Reveals Specific Lipid Peroxidation and Fragmentation Processes: Biogenesis of Pimelic and Azelaic Acid. Plant Physiol. 160: 365-378.

Google Scholar: Author Only Title Only Author and Title

Zuegge, J., Ralph, S., Schmuker, M., McFadden, G.I., and Schneider, G. (2001). Deciphering apicoplast targeting signals - feature extraction from nuclear-encoded precursors of Plasmodium falciparum apicoplast proteins. Gene 280: 19-26.

Google Scholar: Author Only Title Only Author and Title 